\title{
Image Bio-Markers and Gene Expression Data Correlation Framework for Lung Cancer Radio- Genomics Analysis Based on Deep Learning
}

Dong Sui ( $\sim$ suidongcs2016@gmail.com )

Beijing University of Civil Engineering and Architecture https://orcid.org/0000-0002-7887-2111

Maozu Guo

Beijing University of Civil Engineering and Architecture

Xiaoxuan Ma

Beijing University of Civil Engineering and Architecture

Julian Baptiste

University of Maryland Medical Center

Lei Zhang

University of Maryland Baltimore County

Research

Keywords: Radiomics, Radiogenomics, Deep Learning, Genomics Biomarker, GSEA

Posted Date: January 18th, 2021

DOl: https://doi.org/10.21203/rs.3.rs-144196/v1

License: (9) This work is licensed under a Creative Commons Attribution 4.0 International License.

Read Full License 


\section{Image Bio-markers and Gene Expression Data Correlation Framework for Lung Cancer Radio-genomics Analysis Based on Deep Learning}

\author{
Dong Sui ${ }^{1 *}$ \\ , Maozu Guo ${ }^{1}$ \\ , Xiaoxuan $\mathrm{Ma}^{1}$ \\ , Julian Baptiste ${ }^{2}$ \\ and Lei Zhang ${ }^{2}$
}

\begin{abstract}
Background: Precision medicine, a popular treatment strategy, has become increasingly important to the development of targeted therapy. To correlate medical imaging with prognostic and genomic data, researches in radiomics and radiogenomics have provide many pre-defined image features to describe image information quantitatively or qualitatively. However, in previous researches, there are only statistical results which proves high correlation among multi-source medical data, but those can't give intuitive and visual result.
\end{abstract}

Results: In this paper, a deep learning based radio-genomics framework is provided to construct the linkage from lung tumor images to genomics data and implement generation process in turn, which form a bi-direction framework to map multi-source medical data. The imaging features are extracted from auto-encoder under the condition of genomics data. It can obtain much more relevant features than traditional radio-genomics methods. Finally, we use generative adversarial network to transform genomics data onto tumor images, which gives a cogent result to explain the linkage between them.

Conclusions: Our proposed framework provides a deep learning method to do radio-genomics researches more functionally and intuitively.

Keywords: Radiomics; Radiogenomics; Deep Learning; Genomics Biomarker; GSEA;Radiomics; Radio-genomics; Deep Learning; Genomics Bio-markers; GSEA

\section{Background}

Currently, the number of lung cancer patients and cancer-related deaths has occupied a vital position of cancer-related deaths worldwide. Over $70 \%$ of lung cancer patients can only be diagnosed after the onset of symptoms from advanced local or metastatic disease. Unfortunately, the survival rate is only $50 \%$ on the condition that diagnose could be located. It is worst that only less than $20 \%$ of these patients are diagnosed with very early stages. In this context, precision medicine is gaining popularity for providing customized or personalized healthcare, and quantitative imaging has been contributing to significant improvement of the diagnosis procedures [1]. 
In another way, personalized medicine aims to tailor medical care to the individual from view of molecular. High-throughput molecular biology technologies claim to produce biomarkers for disease diagnosis and its prognosis prediction $[2,3,4]$. However, these biospies generated from heterogeneous lesions cannot completely represent the anatomic and physiologic properties, such as the tumor size, anatomic location, and morphology. In another way, image features extracted from these lesions construct a highly informative pathway to disease diagnosis, treatment planning and clinical analysis. Nevertheless, only few studies have constructed radiogenomics frameworks of these information correlations that integrate the genomic and image data $[5,6,7,8]$.

Traditionally, radiology and image-guided interventional therapy have been used to deal with diagnosis and provide anatomical information. However, all these methods must make the patient's physical wounds painful and take longer to heal. To overcome these shortcomings, radiomics extract image features and sub-visual features from radiological images and use state-of-the-art machine learning techniques to provide unique potential for faster and more accurate lung cancer screening. Therefore, by improving the practice of qualitative and quantitative analysis, it is expected to improve the prognosis prediction and response to some treatments. In addition, some radiogenomics researchers have shown that some image features are even associated with genomic changes in tumor DNA [1]. These characteristics can identify specific changes in biological pathways that in turn affect patient management and health outcomes [11, 10].

We develop a radiogenomic framework centered on deep learning (DL) to map image features and genomic data, based on our previous work [12] on the correlation between genomics and images. Conditional autoencoder replace the original model while correlating and more genomic analysis are conducted in this paper. Moreover, inspired by some latent space projection model, we fulfill the transition between tumor image and genomic data.

\section{Radiomics and Radiogenomics Approaches}

Traditionally, radiomics and radiogenomics contain four steps: image acquisition, lesion segmentation, feature extraction and model validation. In relevant researches of radiomics and radiogenomics, features are extracted from medical images qualitatively and quantitatively including semantic (prognostic) and numerical (statistic) features. Then statistical methods will give a correlation between them and genomic data using gene-set enrichment analysis (GSEA). These works improve diagnostic and prognostic performance in various oncologic applications and finally promote the development of precision medicine. Coroller et al. build a correlation between image features and clinical data to predict distant metastasis in lung adenocarcinoma [13]. Abdollahi et al. use statistic features to predict sensorineural hearing loss and get a high accuracy [27]. The correlation between genomic data and images can indicate the linkage of gene change and tumor variation. Aerts et al. provide a quantitative method to correlate gene expression profile data and the low level image features, which can give a decision-support in cancer treatment at low cost [14]. Additionally, Gevaert et al. propose a protocol which maps semantic features and genome data, and get a model with an area under the receiver operating characteristic curve (AUC) of $65 \%$ or greater [29]. Besides these, many researches have proved 
that there is linkage between multi-source medical data. However, the pre-defined features used in these researches are not rich and effective enough, such as the sum of pixels with different percentages. This gives us the thought that mapping these data onto DL methods, which will produce multi-level and rich image features for correlation.

\section{Deep Learning for Radiological Analysis}

Radiomics and DL are focus points in medical imaging field [13]. Radiomics apply image features for prognosis prediction, which is vital due to its clinical significance. DL has been used in medical imaging analysis tasks, such as in CT, MRI, and PET, because its high precision. It can provide informative information about diagnosis, prognostic data, tumor phenotypes, and the gene-protein signatures in lung cancer treatments and prediction [13]. Some recent progresses are as followed.

As a basic model of Convolutional neural networks (CNNs), autoencoder is a popular encoder model. In medical imaging segmentation task, it can give an anatomical prior which eliminates the burden of providing paired example segmentations [21, 19]. As an end-to-end method, autoencoder has been applied for lesion detection and segmentation, pixel repair and prognosis validation [21, 22, 39], which show the features extracted from autoencoder contain mostly image information.

With the similar structure as autoencoder, the U-Net architecture achieves excellent performance on different biomedical applications, including tumor segmentation and CT reconstruction $[17,18]$. As most popular method of segmentation field, U-net shows its robustness and effectiveness, so we will use it to obtain the lesion region.

Recently, There are many applications in several medicals imaging fields using GAN [20] frameworks, including creating translation of label-to-image, mask-toimage or medical cross modality $[24,25,26]$. These generation models can output the images we need according to the input data. However, Conditional Generative Adversarial Nets (CGAN) utilizes extra inputs for fusing more information [23]. It produces more precise result compared to GAN due to the extra condition $y$ in generation process:

$$
\begin{aligned}
& \min _{G} \max _{D} V(D, G)= \\
& \mathbb{E}_{x \sim p_{\text {data }}(x)}[\log D(x \mid y)]+\mathbb{E}_{z \sim p_{z}(z)}[\log (1-D(G(z \mid y)))]
\end{aligned}
$$

It has been applied in many medical fields, including organ segmentation [34], lesion generation [33], etc.. These researchers indicate CGAN architecture can generate expected medical images.

Considering the distribution of images of different categories, Bao J. et al. propose a framework CVAE-GAN [35] based on VAE [36] different from GAN. This model projects images of different categories to different latent space which makes generation of images easy while giving a specific category. Inspired by this work, we will propose similar method to visualize tumors using genomics data. 


\section{Methods}

In this part, we will introduce our methods of the whole framework in Fig. 1 for details. First, an U-net based segmentation method is applied to get TR from original CT image. Then, we use autoencoder to encode the images under the condition of gene. The image features are extracted from different levels of encoder. A series of analysis experiments, including prognosis and GSEA, are applied to these features, prognostic data and genes to prove the correlation among these multi-source data. Finally, a modified CVAE-GAN transforms gene to corresponding TR and give an intuitive result.

\section{Tumor Detection and Segmentation}

In the segmentation stage, we apply U-net model to get cropped tumor images. We input the original CT image to U-net and let it fit its corresponding mask. The architecture of U-Net is displayed in Fig. 2. We choose Dice loss (Eq. 2) here [16] to measure the contact ratio between predicted mask $(P M)$ and ground truth $(G T)$ :

$$
\text { Dice }=\frac{2 \sum_{i}^{N} p_{i} t_{i}}{\sum_{i}^{N} p_{i}+\sum_{i}^{N} t_{i}+\epsilon},
$$

where, $p_{i}$ is the pixel value in $P M, t_{i}$ is the pixel value in $G T, N$ represents total pixels number of an image. And to avoid division by zero, a small constant $\epsilon$ is added to denominator.

\section{Correlation between Gene and Image}

To build the correlation between genes expression data and image features of tumor region in CT image series, we establish a conditional autoencoder to map the distinct source data for keeping the feature extraction ability without missing match. In our previous works, we find that there are two key problems: 1) The dimension of gene data can greatly influent the training effect so that leading to the model collapse. 2) The basic autoencoder can extract multiple level image features. Unfortunately, these features are only obtained without restriction by domain knowledge, so this usually leads to poor correlation between the gene and image feature. In this part, we introduce a gene encoder method for the dimension reduction and a conditional autoencoder for knowledge related feature extraction.

Gene Encoder: The huge dimension of genomic data array is obviously an obstacle to array prediction, which is usually up to $10 \mathrm{k}$. To handle this problem, like the operation in [40] for encoding word vectors, we introduced an encoder for gene data dimension reduction as follows.

Let gene data be a matrix $g_{m n}, m$ is the number of subjects and $n$ is the length of gene array. We use the matrix $w_{n s}$ ( $s$ is objective dimension and is much smaller than $n$ ) to multiply $g_{m n}$ for encoding gene array. The equation is $y=g w$. Then we normalize the encoded gene array $y$ to $[0,1]$ according different gene. Details of the gene encoder is listed in Fig. 4.

Conditional Autoencoder: When the output of encoder $E$ is fitted to encoded gene $y$, the weights of $E$ will be tended to transform image to $y$, but the image information will gradually vanish in this process. To avoid this situation, we add 
the decoder $D$ after the $E$ to fit original image $X$ so that every layer of $E$ can keep image information as much as possible. This process can be formulized as:

$$
\begin{aligned}
& \text { feat, } \tilde{y}=E\left(X ; \theta_{e}\right) \\
& \tilde{X}=D\left(\text { feat } ; \theta_{d}\right)
\end{aligned}
$$

where $\tilde{y}$ is the output of gene encoder, $\theta_{e}$ is weight of $E, \theta_{d}$ is weight of $D$ and feat is middle output of $E$.

Thus, the conditional autoencoder can extract image features which have high correlation with gene data, and simultaneously keep images information. We apply this architecture to map gene and TR. While training the conditional autoencoder, we use perceptual loss [31] and $L_{1,2}$ loss to fit $\tilde{X}$ to $X$ :

$$
L_{\text {feat }}^{\phi, j}(\tilde{X}, X)=\frac{1}{C_{j} H_{j} W_{j}}\left\|\phi_{j}(\tilde{X})-\phi_{j}(X)\right\|_{2}^{2}
$$

where $\phi$ is the pre-trained VGG16 CNN model trained on ImageNet, $j$ is the $j$ th layer in $\phi$, here we use the layer named 'block5_conv3', and $C_{j} H_{j} W_{j}$ is feature map of $j$ th layer. $L_{1,2}$ loss combines the $L_{1}$ loss and $L_{2}$ loss:

$$
L_{1,2}^{\phi}(\tilde{X}, X)=L_{1}(\tilde{X}, X)+L_{2}(\tilde{X}, X) .
$$

We use this $L_{1,2}$ loss to fit $\tilde{Y}$ to $Y$ :

$$
L_{1,2}^{y}(\tilde{Y}, Y)=L_{1}(\tilde{Y}, Y)+L_{2}(\tilde{Y}, Y)
$$

So the final loss function is $L_{C A}$ :

$$
L_{C A}(X, Y)=L_{\text {feat }}^{\phi}(\tilde{X}, X)+L_{1,2}^{\phi}(\tilde{X}, X)+L_{1,2}^{y}(\tilde{Y}, Y)
$$

\section{GCVAE-GAN for Pathological Visualization}

Traditionally, radiogenomic methods can give a mathematic demonstration on the correlation between gene and images, but most of these methods merely provide the relationship numerically without any visual demonstration, and the visual results are greatly demanded for prognosis inferences.

The CVAE-GAN [35] encodes images and categories to Gaussian distribution, then decodes them to original images under the condition of classifier and discriminator. It can generate new images intra category by using noises due to there are many images belong to same category. But for radiogenomics, each tumor region CT image only corresponds to one gene array, which leads to the collapse of generation intra category. Focusing on the problem of data imbalance, we introduce a genomic conditional variational autoencoder GAN (GCAVE-GAN).

Specifically, we change the CVAE-GAN to fitting the tumor images as follows: 1) To solve data limitation, we let each subject belong to different class, and each class contains several different slices of tumor. So our model can generate different TRs by using the interpolation between genes instead of different noises. 2) We apply 
the encoded gene data as categories of corresponding TRs. For magnifying distance of encoded gene data in gene space, we set the threshold value to 0.5 and replace the values of gene with 0 and 1 . Thus, gene data will perform as multi-class labels which control the generation process of model.

See Fig. 4, our model is composed of four parts: 1) An encoder $E$ which projects TRs to latent space $z, 2)$ A generator $G$ which transforms the latent vector $z$ to TR, 3) A discriminator $D$ which judges whether an image is real or fake TR, 4) A classifier $C$ which projects TR to gene space.

To train this model well, there are six loss functions to support whole process [35]. To make the $D$ have strong judgment ability to distinguish real TRs and synthesized TRs, $D$ must minimize the loss function:

$$
L_{D}=-\mathbb{E}_{x \sim P_{r}}[\log D(x)]-\mathbb{E}_{z \sim P_{z}[\log (1-D(G(z)))]} .
$$

To address the problem that the gradient of $G$ is unstable while training, we use the mean feature matching objective proposed in [35]:

$$
L_{G D}=\frac{1}{2}\left\|\mathbb{E}_{x \sim P_{r}} f_{D}(x)-\mathbb{E}_{z \sim P_{z}} f_{D}(G(z))\right\|_{2}^{2},
$$

which acts as perceptual loss, where $f_{D}(x)$ denote features on an first fully connection (FC) layer of the discriminator. It will accelerate the converging speed and improve stability of whole model.

Because the encoded gene data $c$ show the tendency whether a group of genes is overexpressed or underexpressed, we don't fit it using logarithmic loss function. Instead, we apply the mean square loss on it.

$$
L_{C}=\frac{1}{2}\left\|\mathbb{E}_{x \sim P_{r}} C(x)-c\right\|_{2}^{2} .
$$

To make the TR generated by $G$ is belong to corresponding category $c, G$ need to minimize the function

$$
L_{G C}=\frac{1}{2}\left\|\mathbb{E}_{x \sim P_{r}} f_{C}(x)-\mathbb{E}_{z \sim P_{z}} f_{C}(G(z, c))\right\|_{2}^{2} .
$$

The mean feature matching objective is also used here for the same purpose of $L_{G D}$.

For the latent vector in each sample, we adapt the same strategy as [36]. The encoder outputs the mean $\mu$ and covariance $\epsilon$ of latent vector. Then use KullbackLeibler (KL) loss to let distribution of latent space close to gaussian distribution. The $K L$ loss can be formulated as

$$
L_{K L}=\frac{1}{2}\left(\mu^{T} \mu+\sum(\exp (\epsilon)-\epsilon-1)\right) .
$$

Then latent vector $z$ can be sampled as $z=\mu+r \odot \exp (\epsilon)$, where $r N(0, I)$ is a random vector and $\odot$ represents the element-wise multiplication [35]. After $z$ is inputted to generator $G$, we can get the synthetic TR $\tilde{x}$. We combine the $L_{2}$ 
reconstruction loss, perceptual loss and pair-wise feature matching loss to minimize the difference between $x$ and $\tilde{x}$,

$$
\begin{array}{r}
L_{G}=\frac{1}{2}\left(\|x-\tilde{x}\|_{2}^{2}+L_{\text {feat }}^{\phi, j}(x, \tilde{x})\right. \\
+\left\|f_{D}(x)-f_{D}(\tilde{x})\right\|_{2}^{2} \\
\left.+\left\|f_{C}(x)-f_{C}(\tilde{x})\right\|_{2}^{2}\right),
\end{array}
$$

where $L_{\text {feat }}^{\phi, j}$ is defined as Equation $5, f_{D}$ and $f_{C}$ are the features of first FC layer of discriminator $D$ and classifier $C$, respectively.

The final objective function is expressed as:

$$
L=L_{D}+L_{C}+\lambda_{1} L_{K L}+\lambda_{2} L_{G}+\lambda_{3} L_{G D}+\lambda_{4} L_{G C},
$$

where the formulations of each term are defined in Equation 11 16. This model is trained as the original CVAE-GAN and the parameters are $\lambda_{1}=3, \lambda_{2}=1, \lambda_{3}=$ $10^{-3}$ and $\lambda_{4}=10^{-3}$.

\section{Experiments and Results}

In this section, we first conduct three experiments to separately demonstrate the results of models' output in segmentation, correlation and generation stages. Then, we prove the whole framework is comparable with traditional radiogenomics research on statistical analysis results. Finally, we show the generation results on the influence of gene change, and prove that the variance of gene expression will affect tumor states in radiology appearance.

\section{Datasets}

NSCLC Radiogenomics: Non-Small Cell Lung Cancer (NSCLC) is from Cancer Archive [30], where cancerous regions are relabeled as mask by doctors. In this paper, we choose NSCLC to construct our framework and evaluate the linkage among multi-source data. This dataset contains 211 subjects from a NSCLC cohort. Each subject in this dataset includes a CT series which contains hundreds of dicom images. And there are corresponding annotations of each tumor in each subject. Due to these annotations are just a coordinate of tumor, we invite doctors to relabel tumor as mask for us.

\section{ROls Extraction from CT Series}

We separated the NSCLC dataset into training and testing set. The training set has 50 subjects up to 15000 CT images, which contains only image data for TRs segmentation [14]. The testing set has 161 subjects, each of them composed of CT image and its gene expression data. And the these 161 subjects was employed for the gene and image correlation latter. We choose U-net with Dice loss as the detector to recognize the position of tumor. We train it for just 500 epochs using Adam optimizer [32] with a learning rate 0.001. Then it is applied for testing set to detect position of tumor. TR is cropped at a size of $128 \times 128$. The cropped TRs and their corresponding gene data then form the dataset of correlation stage.

Fig. 5 shows the predicted mask of UNet. The pixels belong to tumor are colored in blue which also show the tumor location. These masks cover main part of tumor 
except some irregular edges because of the less obvious edge of tumor, which is precise enough to the detection task. The size and location of tumor can be obtained at the same time. When we crop CT images, some surrounding information, such as bronchial tube and lung edge, which is crucial to mapping stage, are contained for providing more useful information.

\section{Correlation between TRs and Genomic Data}

In this part, we preprocess the gene data to remove the subjects whose gene have not available values. Finally, we get a dataset which has 113 subjects, and each of them contains 6 TRs of one tumor. We randomly sample 90 subjects as training set and 23 subjects as testing set, the ratio of testing set to training set is 1:4. The gene data are encoded using the gene encoder in Section. Then the conditional autoencoder can be trained as a multi-task network. Data augmentation is also employed in training process including horizontal/vertical flip and rotation. We train it using Adam optimizer [32] with a learning rate 0.001 until mean square error has decreased to 0.002 on testing set.

The images that conditional autoencoder outputs are shown in Fig. 6. The similarity between autoencoder's outputs and original TRs indicates model has already extracted images features sufficiently and won't be effected by geometric transformation. Meanwhile, it keeps the correlation between gene and image.

To prove the effectiveness of image features extracted from TRs, we apply hierarchical clustering on gene data and image features. We apply ResNet50 as the encoder of autoencoder. The features of different levels are extracted from last layer of each residual block. Then we apply Locally Linear Embedding (LLE) on them to reduce the dimension of features. Finally, we get four groups of features from different levels for each subject. The numbers of features are 64, 256, 512 and 1024, separately. Then we cluster image features among the samples to prove the correlation between features and disease (shown in Fig. 8). Like the work in [14], we also perform Chi-squared test to verify significant association of the DL features with prognosis data (shown in Table 1).

Also, to prove the effectiveness of condition, we remove it to do ablation experiment. We train the basic autoencoder without condition using the same process and extract features from same position of encoder. Then we calculate the correlation matrix of features (with and without condition) and gene data. Finally, we use box figure (Fig. 7) to show the difference between similarity with and without condition in each level.

To further prove the correlation between gene and image, we do gene-set enrichment analysis using GSEA software [37]. First, the Pearson correlation coefficient matrix $c$ is calculated by features $f$ and gene $g$. In each level, 10 features are selected according to $s$, the sum of all correlation coefficient's absolute value of each feature. Then genes will be sorted according to $s$ to form pre-ranked files $l$, which are input of GSEA pre-ranked analysis. GSEA will find whether there is a gene set with known function appears on the top or bottom of $l$, which indicates features have positive or negative effect on that gene set. Finally, we will get the false discovery rate (FDR) and normalized enrichment score (NES) of each found gene set. The whole analysis process is expressed as Algorithm 1 and the correlation result is showed in Fig. 9. 
In this part, we construct a linkage between DL features and genomic data from image to gene and prognosis domain, the results demonstrate this linkage has the same effectiveness as it in traditional radiology features, in addition, our proposed linkage can give an multilevel results unlike the handcrafted features. DL features work as traditional radiology features in this stage and the results of GSEA and prognosis validation are convincing.

\section{TRs Generation under Gene Guidance}

In this part, gene data are transformed to binary arrays which are called as controllers. Our task is to visualize TR using the corresponding controller. Each controller is the sum of all gene expression values weighted by different coefficients. We train this model for $4 \mathrm{k}$ epochs and get the cogent results.

The encoder of model first encodes gene and TRs to latent space, which is very close to the gaussian space. To prove the continuity of model when it generates TR, we use interpolation between two controllers to generate new TR whose state falls in between two corresponding TRs. Unlike the linear interpolation, we switch the state of controller one by one as our "controller interpolation", because value of controller only presents gene is overexpressed or underexpressed (0 or 1). The TRs generated by this method are showed in Fig. 10. We can find that generated images are changing gradually and continuously with controllers switched.

The generator then concatenates gene and a noise $z$ to form an array and raise its dimension for generation process. We use the t-distributed stochastic neighbor embedding (TSNE) to visualize these arrays in latent space, part of which is shown in Fig. 11. We can observe that tumors with similar characteristics are projected closely in the latent space. Tumors in Group 1 are close to right edge of right lung and tumors in Group 2 are close to front edge of lung. Given this result, we will find the common ground of these similar tumors during generation process and how these controllers affect generation of tumors.

We select several groups of tumors, mainly about location and size. The controllers with activated state (whose value $=1$ ) in every sample of a group are extracted as the characteristic controllers. To research location controller, as a control, a template tumor is generated using a random noise $z$ and a zero-like array which indicates all controllers are frozen. Then we activate the location controllers (including front, behind, left and right side) and use them to generate tumors (shown in Fig. 12 row 1 ). An enhancement can be seen in corresponding location, which means tumor is closer to thorax. To further prove the ability of location controllers, we freeze them while generating tumors with corresponding lung edge (shown in Fig. 13 (a,b,c,d)). We can easily find that when the corresponding controllers are frozen, outline of lung losses on some level in the corresponding position, which means tumor is far away from thorax.

We apply similar method when research size controller. They are activated one by one while generating tumors (shown in Fig. 12 row 2). The template tumor is bigger and bigger with size controllers activated gradually. To further prove the ability of size controllers, we freeze them while generating big tumors and activate them while generating small tumors. The results are shown in Fig. 13 (e,f), which indicates strong correlation between these controllers and tumor size. 
In this part, we also link the genomic data and corresponding TRs. From the direction of gene raw data to TR images, we build a GCVAE-GAN model for this transformation. Considering the generation ability of model and the limitation of data, results of interpolation experiment shows that the model is continuous (see Fig. 10). In addition, we also change the gene expression values manually in a reasonable way. We validate the proposed framework by using the direction from genomic data to TRs. By this way, we use interpolation method to generate new TRs and change gene expression values to observe the variation of TR. The results prove that there is strong linkage from genome data to CT images.

\section{Discussion}

[14] proved that the genomic data and image computational features have strongly correlation with each other. We also demonstrate this result by using the proposed framework. And it will provide a new noninvasive way of investigating phenotypic information as traditional radiogenomics.

After bi-direction validation(gene to image and image to gene), we find that some gene sets have high NES while correlated with DL features, among which, most gene sets are associated with cancer directly or indirectly by regulating cell activity (see Table 2). We cluster the samples into several distinct groups by using the deep features. Putting aside its result verification, we perform a statistical analysis on these cluster results and the prognostic data (T-stage and histology). The p_value of Chi-squared test is low (which is $\leq 0.05$ ) between them, see Table 1 . This results demonstrate that our proposed framework can fulfill the correlation task all by using multi-levels deep features, especially on the correlation of tumor types and stages, that is the same as traditional methods [14].

The previous work [38] has demonstrated that the location of tumor has strong correlation with specific metagenes. In this context, we extent these anatomy information by some parameters, such as tumor location with thorax and tumor size. In this way, we find these genes as controllers that can guide the tumor growth. Different controller will enhance pixel values of corresponding part, which implicates there are some relations between gene and tumors. Due to each controller is consist of all gene values with different weights, an activated controller indicates high expression value of genes with high weights.

We change the location controllers and find that tumor will be away from thorax when froze the corresponding controllers (see Fig. 13(a, b, c, d)). In another way, tumor size is an important information in its prognosis and it can reflect the tumor states and chemoradiotherapy. We investigated the tumor size related genes sets and find that these size controllers have strong correlations with tumor growth. In our framework, we attempt to transform the tumor anatomy structure size by related genes, and the results indicate that the size controllers can enlarge and shrink the tumors when activated or frozen without disturbing other part of tumor region (see Fig. $13(\mathrm{e}, \mathrm{f}))$.

Advantages: Radiogenomics researches with handcrafted features are obscure and have no anatomical significance for clinic doctors. Furthermore, most of these features are redundant and strongly depend on domain knowledge. Unlike that, our proposed framework can get as many features as we need which are conditioned 
by genes. Highly gene associated features are easy to be filtered through similarity matrix between gene and features. Then it is easy to build an effective correlation between genomic and radiology information by these hierarchical features.

Benefiting by this integrated dataset which has all data needed in radiogenomic researches, including original CT images, genomic and prognosis data, we can find and study the linkage between image and gene. This framework provides a method to find specific gene-set which has high correlation with specific DL features and gene which has high correlation with needed trait of TRs.

Limitations: Because of limitations relevant to the dataset scale and unbalance issues, our visualization results show that there are some abnormal instances because of lung edge and other organ like heart. The model can't ignore this obvious trait to pay attention to others. There would be more controllers which control other traits of tumor with a large dataset which is classified by tumor location. But in our work, these controllers are not completely independent because of the coupling phenomenon in tumor generation. Different groups of controllers influent each other and will change other traits of tumor.

As for the proposed framework has showed its capability in mapping the genomic data and tumor images, we will focus on dataset enlargement and combining different dataset from different sources and data categories.

\section{Conclusion}

In this paper, we proposed a radiogenomics analysis framework based on DL, through which, we could accomplish most analysis radiogenomic tasks. Moreover, based on prognosis analysis, we could establish a preliminary visual representation of the cancer growth in its later stages. We validated the framework bi-direction from gene to image and image to gene respectively. From image to gene, the statistical results (see Table 1) prove that the proposed framework is consistent with the traditional radiogenomic analysis results, and the differentially expressed genes could be screened out through multi-level features in the networks, which is better than traditional radiogenomic methods. From genes to image, the results validate that gene expression differences can influent tumor growth, which reversely shows the association between two domains. Our future work mainly focuses on the following aspects: 1) Finding more effective method to encode gene for keeping genomic data information as much as possible. 2) Not limited to lung cancer, we will do more research on other cancer types using our framework. 3) We will construct correlation between multi-modal image (including CT, MRI and pathological image) and genomic data. 4) The generation method with more similar dataset is under our researching.

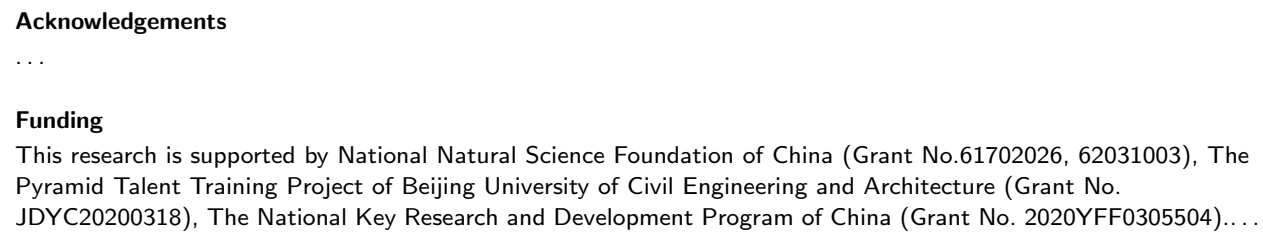




\section{Authors' contributions}

Dong Sui have made substantial contributions to conception, design, acquisition of data, analysis and interpretation of data, he agree to be accountable for all aspects of the work in ensuring that questions related to the accuracy or integrity of any part of the work are appropriately investigated and resolved. Julian Baptiste and Lei Zhang have been involved in drafting the manuscript or revising it critically for important intellectual content. Xiaoxuan Ma and Maozu Guo have given final approval of the version to be published.

Availability of data and materials

The image and genomics dataset are downloaded from The Cancer Imaging Archive, which is a public dataset for cancer detection and analysis method development. we choose NSCLC-Radiomics-Genomics dataset contains images from 89 non-small cell lung cancer (NSCLC) patients that were treated with surgery. For these patients pretreatment CT scans, gene expression, and clinical data are available. This dataset refers to the Lung3 dataset of the study published in Nature Communications.

URL:https://www.cancerimagingarchive.net/ ...

Ethics approval and consent to participate

This manuscript has no ethics problems...

Competing interests

No.

Consent for publication

Yes....

Authors' information

Text for this section. .

Author details

${ }^{1}$ School of Electrical and Information Engineering, Beijing University of Civil Engineering and Architecture, Beijing,

China. ${ }^{2}$ Diagnostic Radiology and Nuclear Medicine, University of Maryland, Baltimore, MD, USA.

\section{References}

1. Thawani R, McLane M, Beig N, et al. Radiomics and radiogenomics in lung cancer: A review for the clinician[J]. Lung cancer (Amsterdam, Netherlands), 2018, 115: 34.

2. Sotiriou C. Molecular biology in oncology and its influence on clinical practice: gene expression profling [abstr]. Ann Oncol 2009;20(Suppl 4):v10.

3. Pao W, Kris MG, lafrate AJ, et al. Integration of molecular profling into the lung cancer clinic. Clin Cancer Res 2009;15(17):5317-5322.

4. Gevaert O, De Moor B. Prediction of cancer outcome using DNA microarray technology: past, present and future. Expert Opin Med Diagn 2009;3(2):157-165.

5. Segal E, Sirlin CB, Ooi C, et al. Decoding global gene expression programs in liver cancer by noninvasive imaging. Nat Biotechnol 2007;25(6):675-680.

6. Diehn M, Nardini C, Wang DS, et al. Identifcation of noninvasive imaging surrogates for brain tumor gene-expression modules. Proc Natl Acad Sci U S A 2008;105(13):5213-5218.

7. Kuo MD, Gollub J, Sirlin CB, Ooi C, Chen X. Radiogenomic analysis to identify imaging phenotypes associated with drug response gene expression programs in hepatocellular carcinoma. J Vasc Interv Radiol 2007;18(7): 821-831.

8. Rutman AM, Kuo MD. Radiogenomics: creating a link between molecular diagnostics and diagnostic imaging. Eur J Radiol 2009;70(2): 232-241.

9. Fitzmaurice C, Allen C, Barber R M, et al. Global, regional, and national cancer incidence, mortality, years of life lost, years lived with disability, and disability-adjusted life-years for 32 cancer groups, 1990 to 2015: a systematic analysis for the global burden of disease study[J]. JAMA oncology, 2017, 3(4): 524-548.

10. Zhuo $\mathrm{Y}$, Feng $\mathrm{M}$, Yang $\mathrm{S}$, et al. Radiomics nomograms of tumors and peritumoral regions for the preoperative prediction of spread through air spaces in lung adenocarcinoma[J]. Translational oncology, 2020, 13(10):100820.

11. Giger M L, Karssemeijer N, Schnabel J A. Breast image analysis for risk assessment, detection, diagnosis, and treatment of cancer.[J]. Annual Review of Biomedical Engineering, 2013, 15(1):327-357.

12. Li S, Han H, Sui D, et al. A Novel Radiogenomics Framework for Genomic and Image Feature Correlation using Deep Learning[C]. 2018 IEEE International Conference on Bioinformatics and Biomedicine (BIBM). IEEE, 2018: 899-906.

13. Coroller T P, Grossmann $\mathrm{P}$, Hou $\mathrm{Y}$, et al. CT-based radiomic signature predicts distant metastasis in lung adenocarcinoma[J]. Radiotherapy \& Oncology, 2015, 114(3):345-350.

14. Aerts H J W L, Velazquez E R, Leijenaar R T H, et al. Decoding tumour phenotype by noninvasive imaging using a quantitative radiomics approach[J]. Nature Communications, 2014, 5:4006.

15. Milletari F, Navab N, Ahmadi S A. V-Net: Fully Convolutional Neural Networks for Volumetric Medical Image Segmentation[J]. 2016:565-571.

16. Ronneberger O, Fischer P, Brox T. U-Net: Convolutional Networks for Biomedical Image Segmentation[C]. International Conference on Medical Image Computing and Computer-Assisted Intervention. Springer, Cham, 2015:234-241.

17. Dong H, Yang G, Liu F, et al. Automatic Brain Tumor Detection and Segmentation Using U-Net Based Fully Convolutional Networks[J]. 2017:506-517.

18. Han Y S, Yoo J, Ye J C. Deep Residual Learning for Compressed Sensing CT Reconstruction via Persistent Homology Analysis[J]. arXiv preprint arXiv:1611.06391, 2016. 
19. Dalca A V, Guttag J, Sabuncu M R. Anatomical Priors in Convolutional Networks for Unsupervised Biomedical Segmentation[C]. Proceedings of the IEEE Conference on Computer Vision and Pattern Recognition. 2018: 9290-9299.

20. Goodfellow I J, Pougetabadie J, Mirza M, et al. Generative Adversarial Networks[J]. Advances in Neural Information Processing Systems, 2014, 3:2672-2680.

21. Xu J, Xiang L, Liu Q, et al. Stacked Sparse Autoencoder (SSAE) for Nuclei Detection on Breast Cancer Histopathology Images[J]. IEEE Trans Med Imaging, 2016, 35(1):119-130.

22. Gondara L. Medical image denoising using convolutional denoising autoencoders[J]. 2016:241-246.arXiv preprint arXiv:1608.04667

23. Mirza M, Osindero S. Conditional Generative Adversarial Nets[J]. Computer Science, 2014:2672-2680.

24. Nie D, Trullo R, Lian J, et al. Medical image synthesis with context-aware generative adversarial networks[C] International Conference on Medical Image Computing and Computer-Assisted Intervention. Springer, Cham, 2017: 417-425

25. Schlegl T, Seeböck P, Waldstein S M, et al. Unsupervised Anomaly Detection with Generative Adversarial Networks to Guide Marker Discovery[J]. 2017:146-157.

26. Ben-Cohen A, Klang E, Raskin S P, et al. Virtual PET Images from CT Data Using Deep Convolutional Networks: Initial Results[J]. 2017:49-57.

27. Abdollahi H, Mostafaei S, Cheraghi S, et al. Cochlea CT radiomics predicts chemoradiotherapy induced sensorineural hearing loss in head and neck cancer patients: a machine learning and multi-variable modelling study[J]. Physica Medica, 2018, 45: 192-197.

28. Wang K, Lu X, Zhou H, et al. Deep learning Radiomics of shear wave elastography significantly improved diagnostic performance for assessing liver fibrosis in chronic hepatitis B: a prospective multicentre study[J]. Gut, 2018: gutjnl-2018-316204.

29. Gevaert $\mathrm{O}, \mathrm{Xu} \mathrm{J}$, Hoang $\mathrm{C} \mathrm{D}$, et al. Non-small cell lung cancer: identifying prognostic imaging biomarkers by leveraging public gene expression microarray dataj ${ }^{a}$ methods and preliminary results[J]. Radiology, 2012, 264(2) 387-396.

30. Bakr, Shaimaa; Gevaert, Olivier; Echegaray, Sebastian; Ayers, Kelsey; Zhou, Mu; Shafiq, Majid; Zheng, Hong; Zhang, Weiruo; Leung, Ann; Kadoch, Michael; Shrager, Joseph; Quon, Andrew; Rubin, Daniel; Plevritis, Sylvia; Napel, Sandy.(2017). Data for NSCLC Radiogenomics Collection. The Cancer Imaging Archive.

31. Johnson J, Alahi A, Fei-Fei L. Perceptual losses for real-time style transfer and super-resolution[C]. European Conference on Computer Vision. Springer, Cham, 2016: 694-711.

32. Kingma D P, Ba J. Adam: A method for stochastic optimization[J]. arXiv preprint arXiv:1412.6980, 2014.

33. Jin D, Xu Z, Tang Y, et al. CT-Realistic Lung Nodule Simulation from 3D Conditional Generative Adversarial Networks for Robust Lung Segmentation[J]. 2018.

34. Dong S, Luo G, Wang K, et al. VoxelAtlasGAN: 3D Left Ventricle Segmentation on Echocardiography with Atlas Guided Generation and Voxel-to-voxel Discrimination[J]. arXiv preprint arXiv:1806.03619, 2018.

35. Bao J, Chen D, Wen F, et al. CVAE-GAN: fine-grained image generation through asymmetric training[J] CoRR, abs/1703.10155, 2017, 5.

36. Larsen A B L, Sønderby S K, Larochelle H, et al. Autoencoding beyond pixels using a learned similarity metric[J]. arXiv preprint arXiv:1512.09300, 2015

37. Subramanian A, Tamayo P, Mootha V K, et al. Gene set enrichment analysis: a knowledge-based approach for interpreting genome-wide expression profiles[J]. Proceedings of the National Academy of Sciences, 2005 102(43): 15545-15550

38. Zhou M, Leung A, Echegaray S, et al. Non-Small Cell Lung Cancer Radiogenomics Map Identifies Relationships between Molecular and Imaging Phenotypes with Prognostic Implications.[J]. Radiology, 2017, 286(1):161845

39. Lao J, Chen Y, Li Z C, et al. A deep learning-based radiomics model for prediction of survival in glioblastoma multiforme[J]. Scientific reports, 2017, 7(1): 10353.

40. $\mathrm{Xu} \mathrm{T}$, Zhang $\mathrm{P}$, Huang $\mathrm{Q}$, et al. Attngan: Fine-grained text to image generation with attentional generative adversarial networks[C]. Proceedings of the IEEE Conference on Computer Vision and Pattern Recognition. 2018: 1316-1324.

Figures

Figure 1: Work flow of our proposed framework. Stage 1: Tumor segmentation. Stage 2: Gene and image mapping. Stage 3: Synthetic tumor region generation.

Figure 2: U-net architecture for tumor segmentation.

Tables

Algorithms 
Figure 3: Illustration of GCVAE-GAN architecture. For better demonstrating this model, it is shown in 3D view. The erect labels are names of different modules and the italicized are operations between modules. The direction of arrows indicates the flow of data. Each sub-model is shown in different axis with name on the left $(E, G$, $D, C)$ and all six losses used in this model are connected by outputs of model and expectation.

Figure 4: Illustration of GCVAE-GAN architecture. For better demonstrating this model, it is shown in 3D view. The erect labels are names of different modules and the italicized are operations between modules. The direction of arrows indicates the flow of data. Each sub-model is shown in different axis with name on the left (E, G, $D, C)$ and all six losses used in this model are connected by outputs of model and expectation.

Figure 5: Illustration of our conditional autoencoder architecture. Images in row a are original CT images, tumors are labeled in red boundingboxes and the blue masks are results of segmentation. Images in row $\mathbf{b}$ are cropped TRs with size of $128 \times 128$.

Figure 6: The tumor images outputted by decoder in test set. Images in the row a are different original TRs, and images in row $\mathbf{b}$ are corresponding synthetic TRs.

Figure 7: The difference of similarity with and without condition in each level. The similarity with condition is obviously higher than that without condition except level 1.

Figure 8: The difference of similarity with and without condition in each level. The similarity with condition is obviously higher than that without condition except level 1.

Figure 9: The correlation matrix between top ten DL features (level 4) related with all gene in each level and gene sets with high NES (NES $\geq 0.25$ ).

Figure 10: Tumor generation process while changing controllers gradually. The different rows are different samples. The first and last image in each row is real TR, and images between then are generated by "controller interpolation". 
Figure 11: Part of samples' distribution on TSNE space. The numbers are index of samples. The samples in group 1 are all close to right edge of lung and those in group 2 are close to front of lung.

Figure 12: Tumors generated using different location controllers (row a) and size controllers (row b). In row a, The first one a- 1 is the template, the others are tumors generated using activated front, behind, left, right controllers, respectively. In row b, The first one b- 1 is also the template, the others are generated with size controllers activated gradually.

Figure 13: TRs generated by GCVAE-GAN with controllers activated or frozen. The images of group (a to d) are generated with corresponding controllers (front, behind, left, right) frozen. The images of group $(e, f)$ are generated with size controllers activated and frozen, respectively. In each group, images of first row are real TRs, images in second row are generated by corresponding genes which are controllers here, and images in third row are generated by corresponding switched controllers (frozen in (e) and activated in others).

Table 1: $\mathrm{P}$ _values of Chi-squared test between features and prognosis.

\begin{tabular}{c|cccc}
\hline P-value & T-stage & N-stage & M-stage & Histology \\
\hline Level1 & 0.0775848 & 0.0073137 & 0.8742585 & 0.4826525 \\
Level2 & 0.0000462 & 0.0686717 & 0.2358527 & 0.0012095 \\
Level3 & 0.0013855 & 0.1856763 & 0.1745525 & 0.0097451 \\
Level4 & 0.0012979 & 0.0603604 & 0.0541809 & 0.0258452 \\
\hline
\end{tabular}

Table 2: Some high NES gene sets with correlated with multi-level DL features. $\checkmark$ means this gene set has high NES associated with corresponding DL features, and opposite for $\times$. In addition, all of these gene sets all have strong association with cancer directly and indirectly.

\begin{tabular}{c|cccc}
\hline Gene Set / Level & Level1 & Level2 & Level3 & Level4 \\
\hline ALLOGRAFT REJECTION & $\checkmark$ & $\checkmark$ & $\times$ & $\checkmark$ \\
MITOTIC SPINDLE & $\checkmark$ & $\checkmark$ & $\checkmark$ & $\times$ \\
DNA REPAIR & $\checkmark$ & $\checkmark$ & $\times$ & $\checkmark$ \\
KRAS SIGNALING UP & $\checkmark$ & $\times$ & $\checkmark$ & $\times$ \\
KRAS SIGNALING DOWN & $\times$ & $\checkmark$ & $\checkmark$ & $\times$ \\
MYC TARGETS V1 & $\checkmark$ & $\checkmark$ & $\times$ & $\checkmark$ \\
MYC TARGETS V2 & $\checkmark$ & $\checkmark$ & $\times$ & $\checkmark$ \\
COMPLEMENT & $\checkmark$ & $\checkmark$ & $\times$ & $\checkmark$ \\
TNFA SIGNALING VIA NFKB & $\checkmark$ & $\checkmark$ & $\times$ & $\checkmark$ \\
IL6 JAK STAT3 SIGNALING & $\checkmark$ & $\times$ & $\checkmark$ & $\times$ \\
E2F TARGETS & $\checkmark$ & $\checkmark$ & $\times$ & $\checkmark$ \\
TGF BETA SIGNALING & $\checkmark$ & $\checkmark$ & $\checkmark$ & $\times$ \\
IL2 STAT5 SIGNALING & $\checkmark$ & $\times$ & $\times$ & $\checkmark$ \\
P53 PATHWAY & $\checkmark$ & $\checkmark$ & $\times$ & $\checkmark$ \\
EPITHELIAL MESENCHYMAL TRANSITION & $\checkmark$ & $\checkmark$ & $\times$ & $\checkmark$ \\
\hline
\end{tabular}




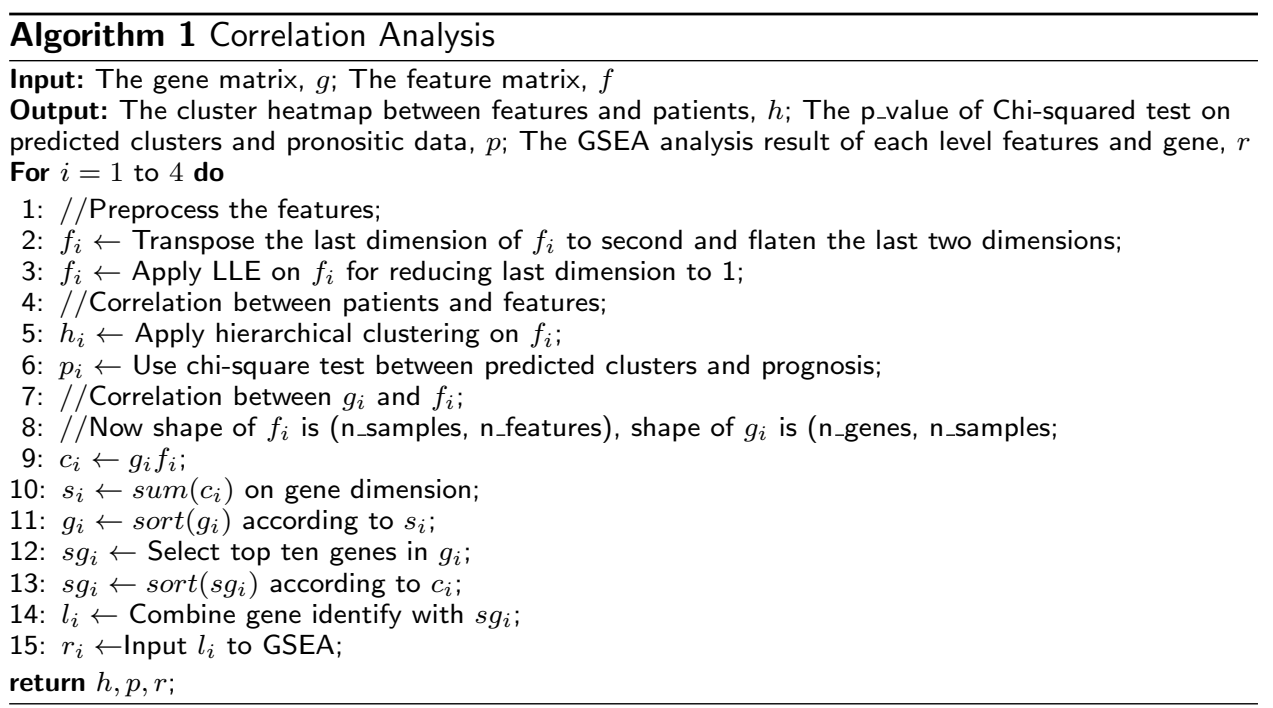


Stage 1

Skip - connection $(S)$

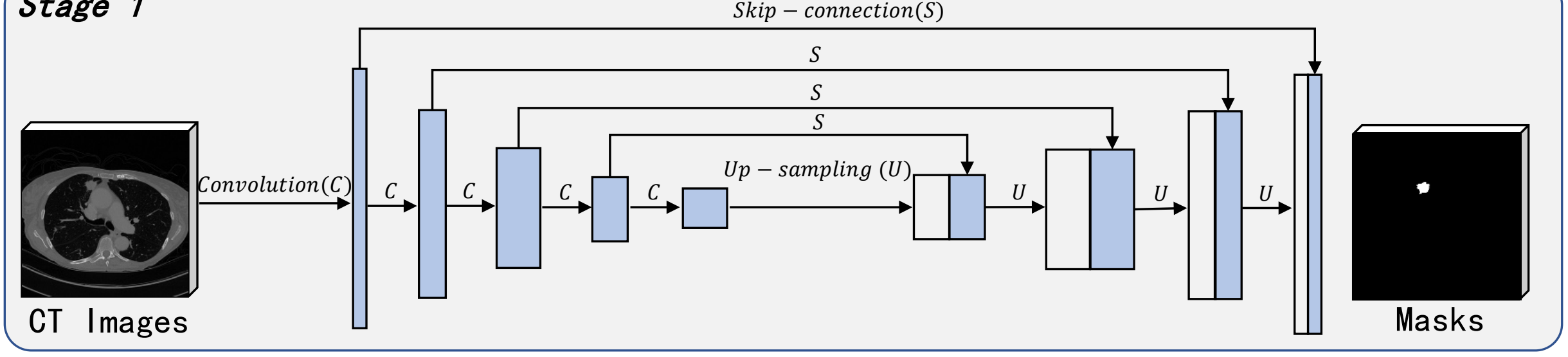




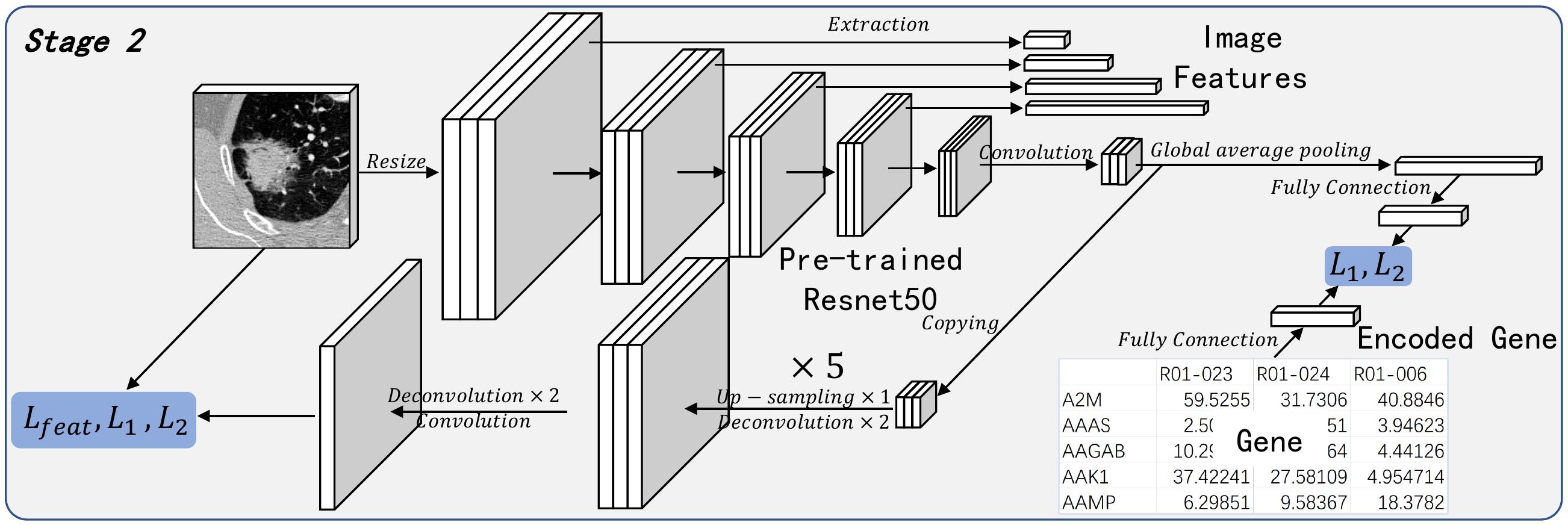




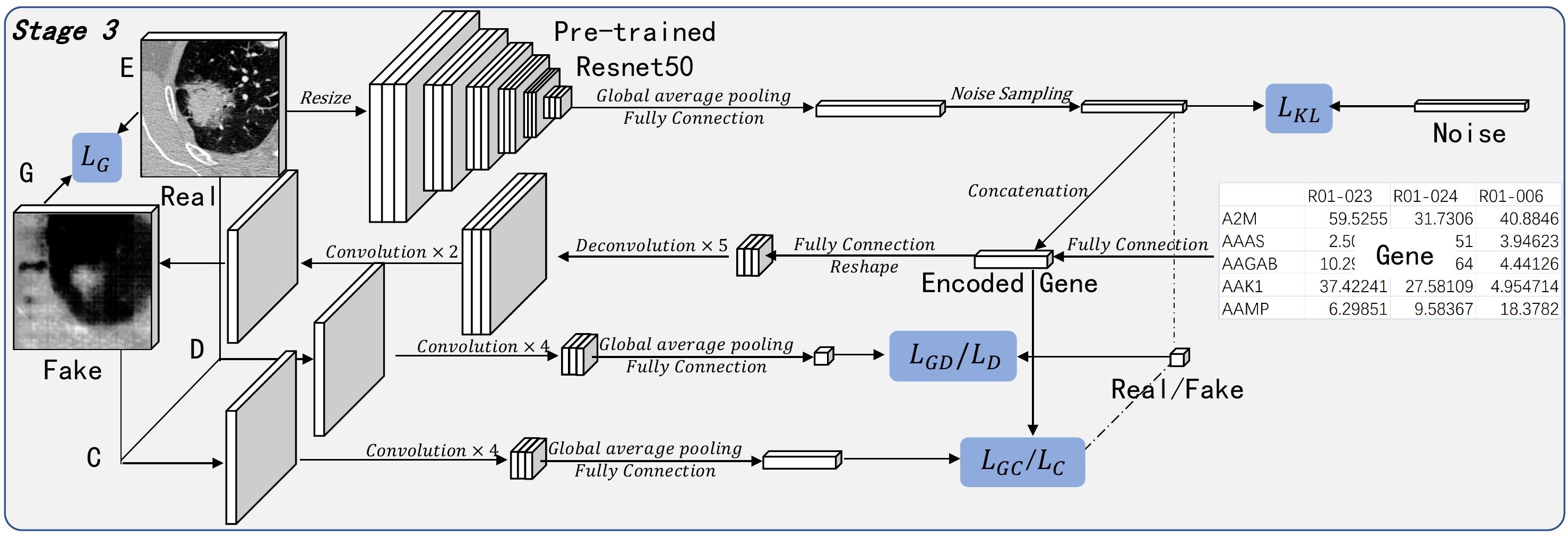




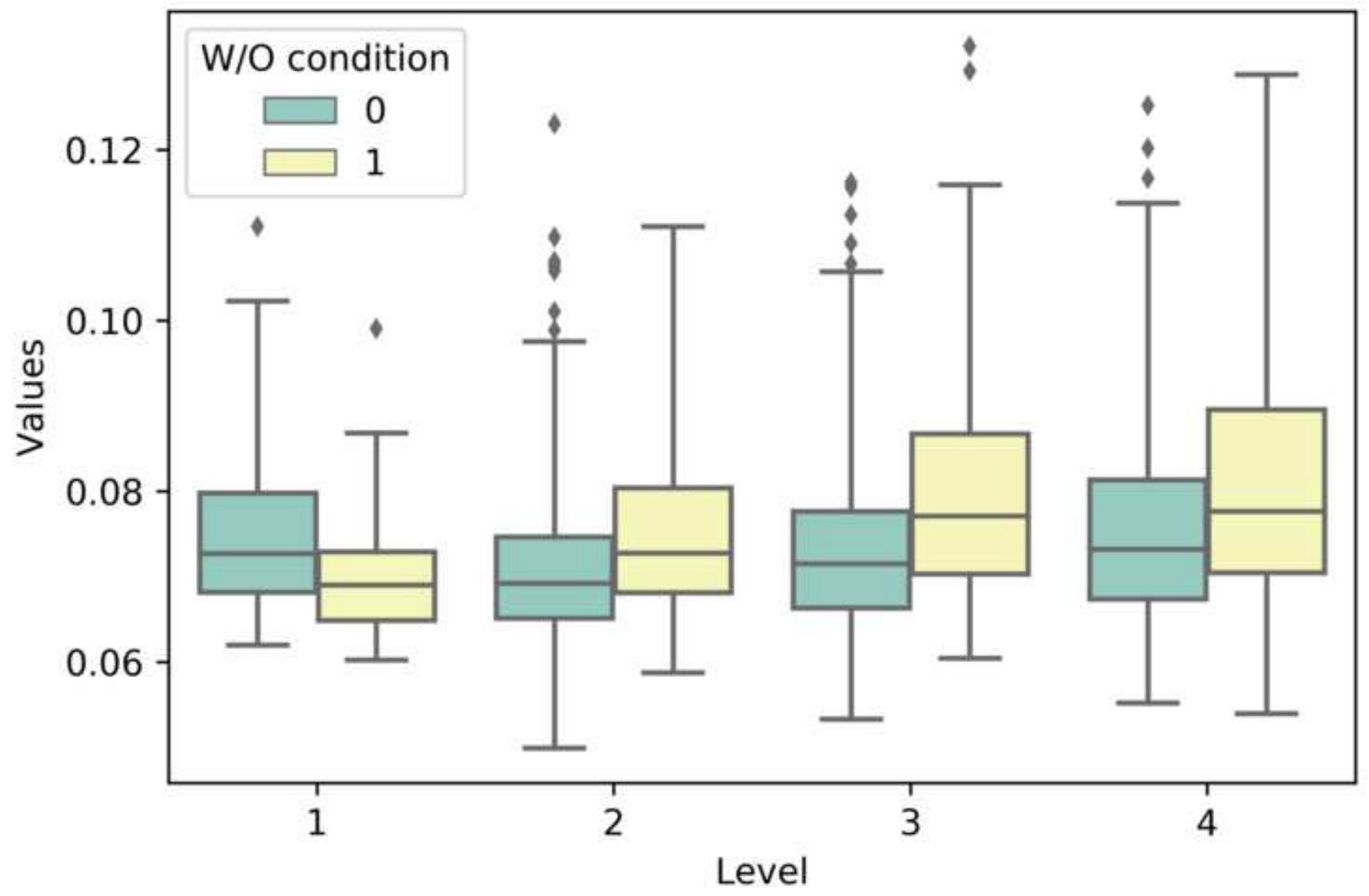




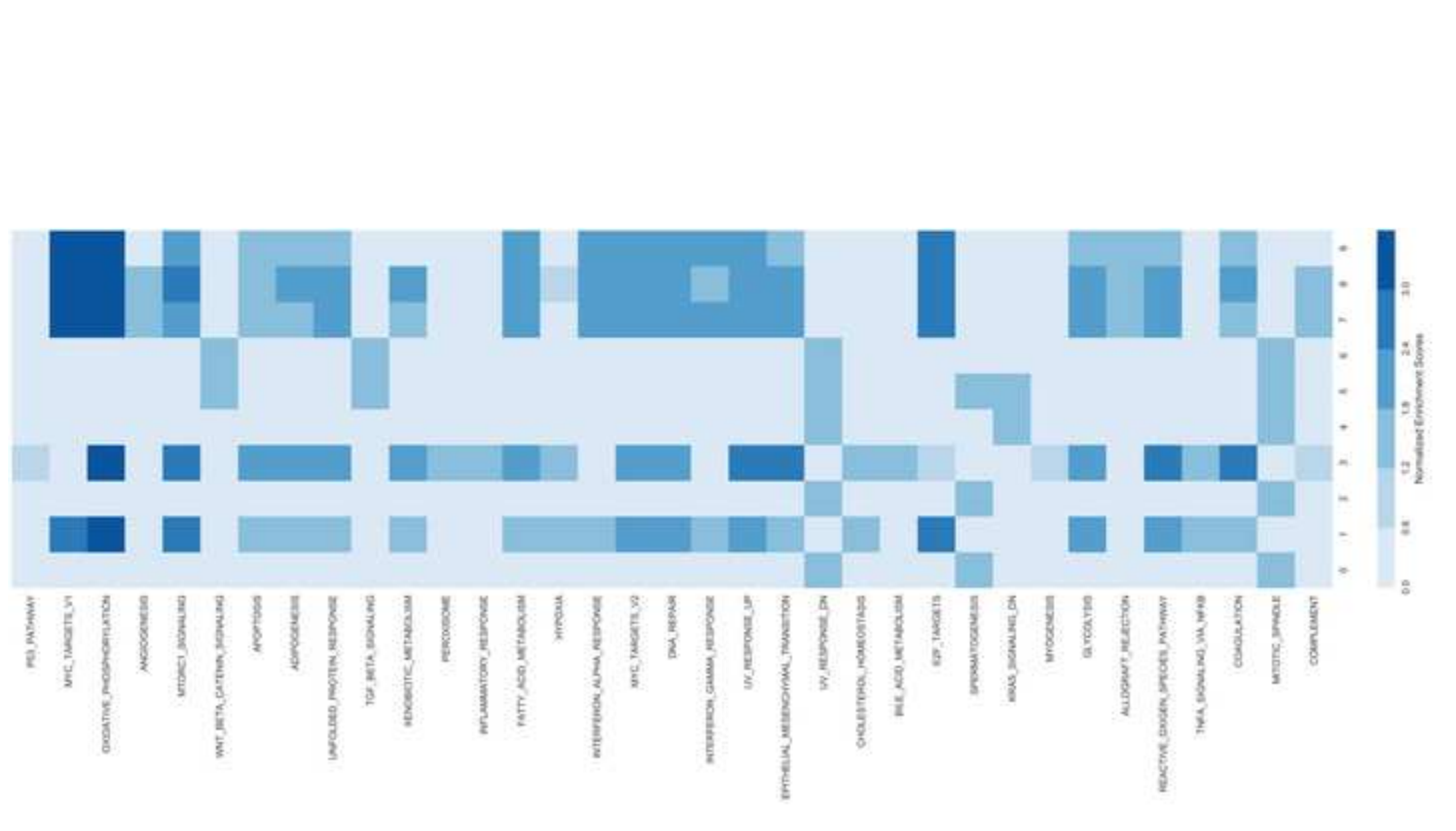

.
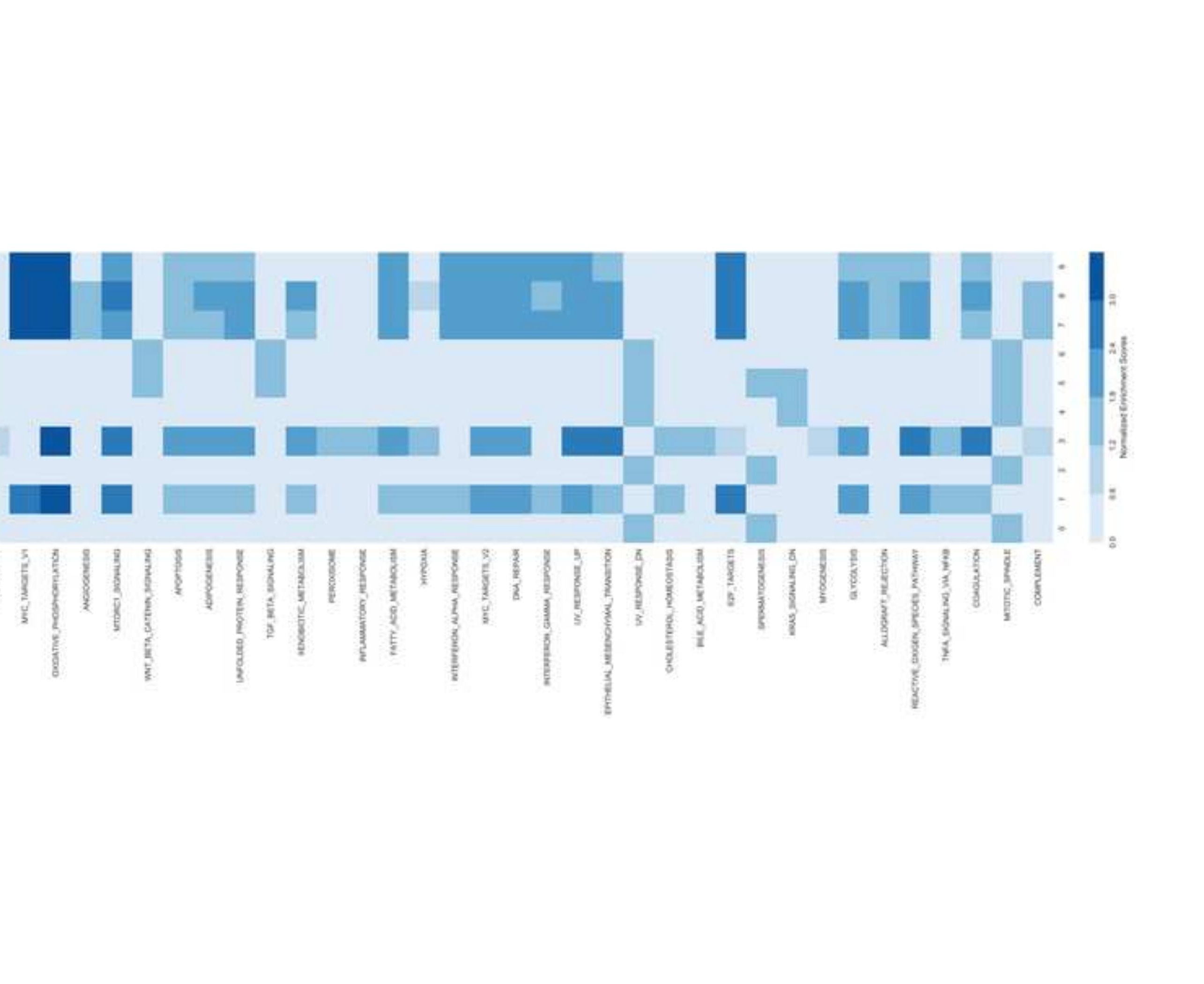


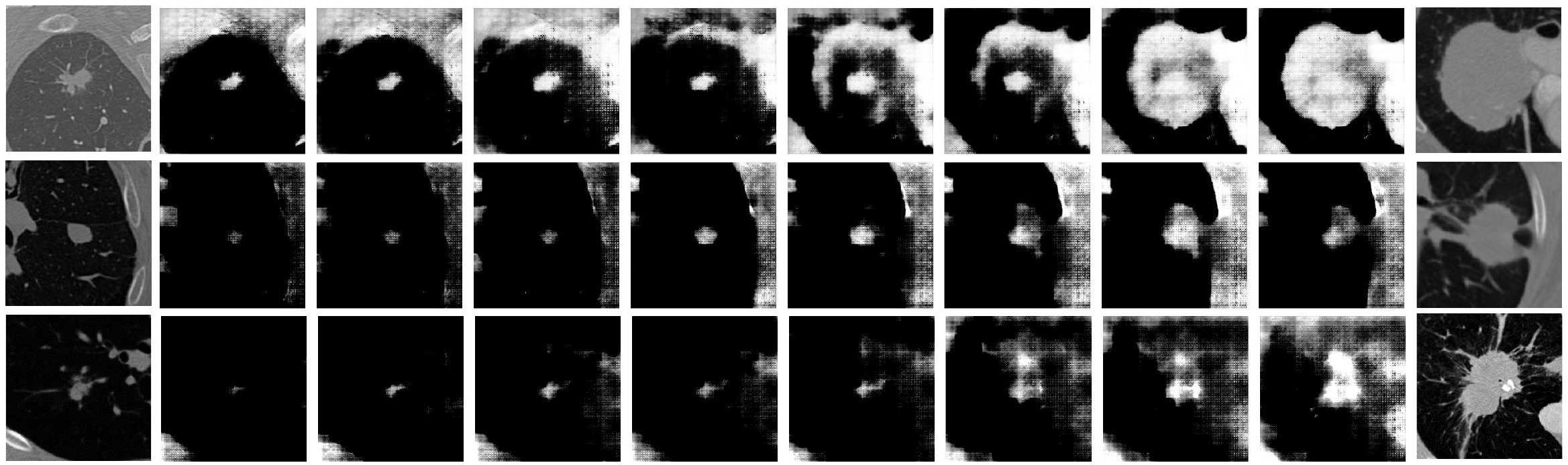




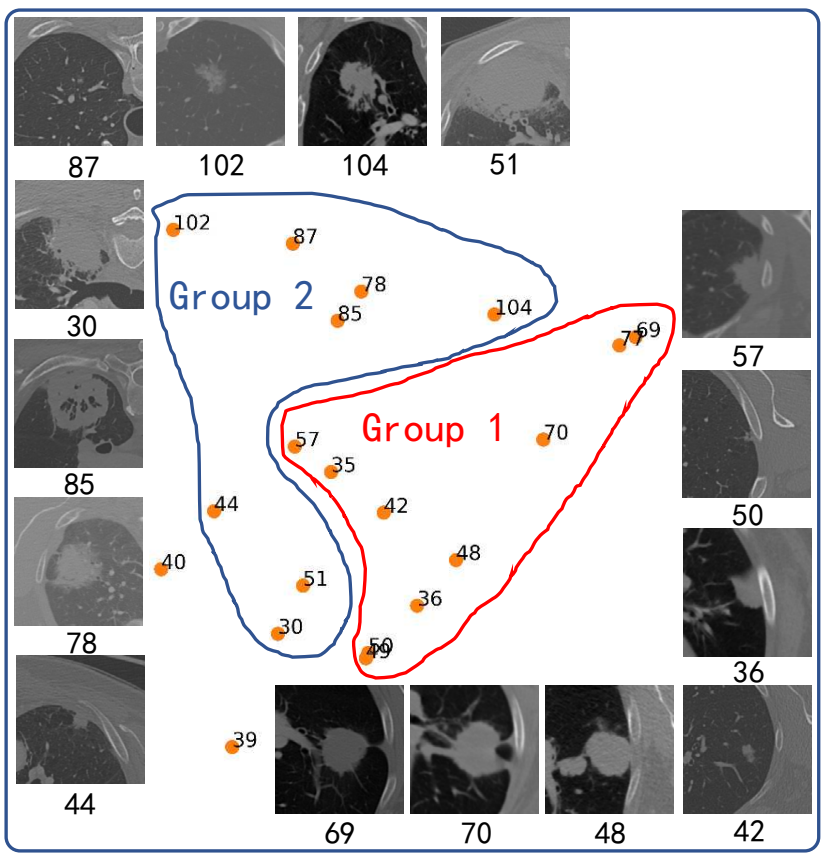




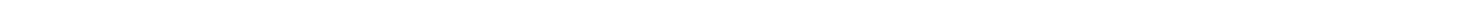




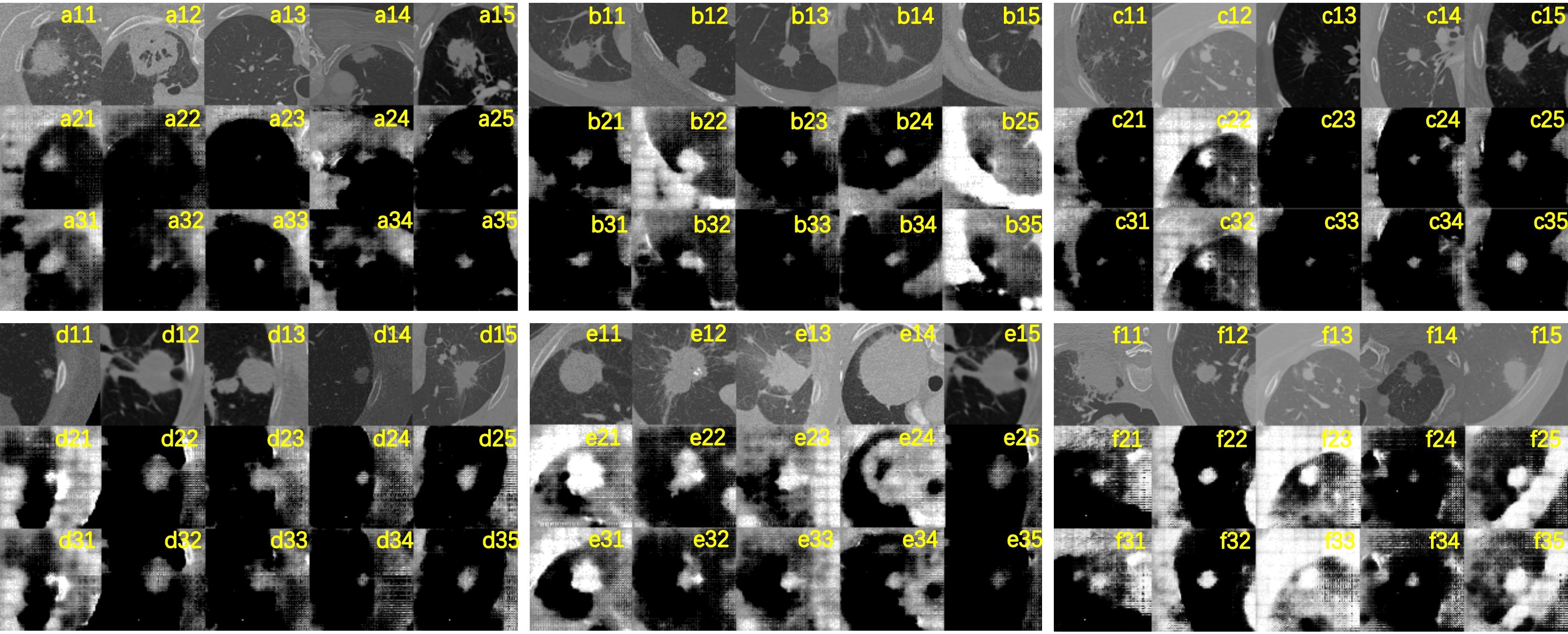


Figures

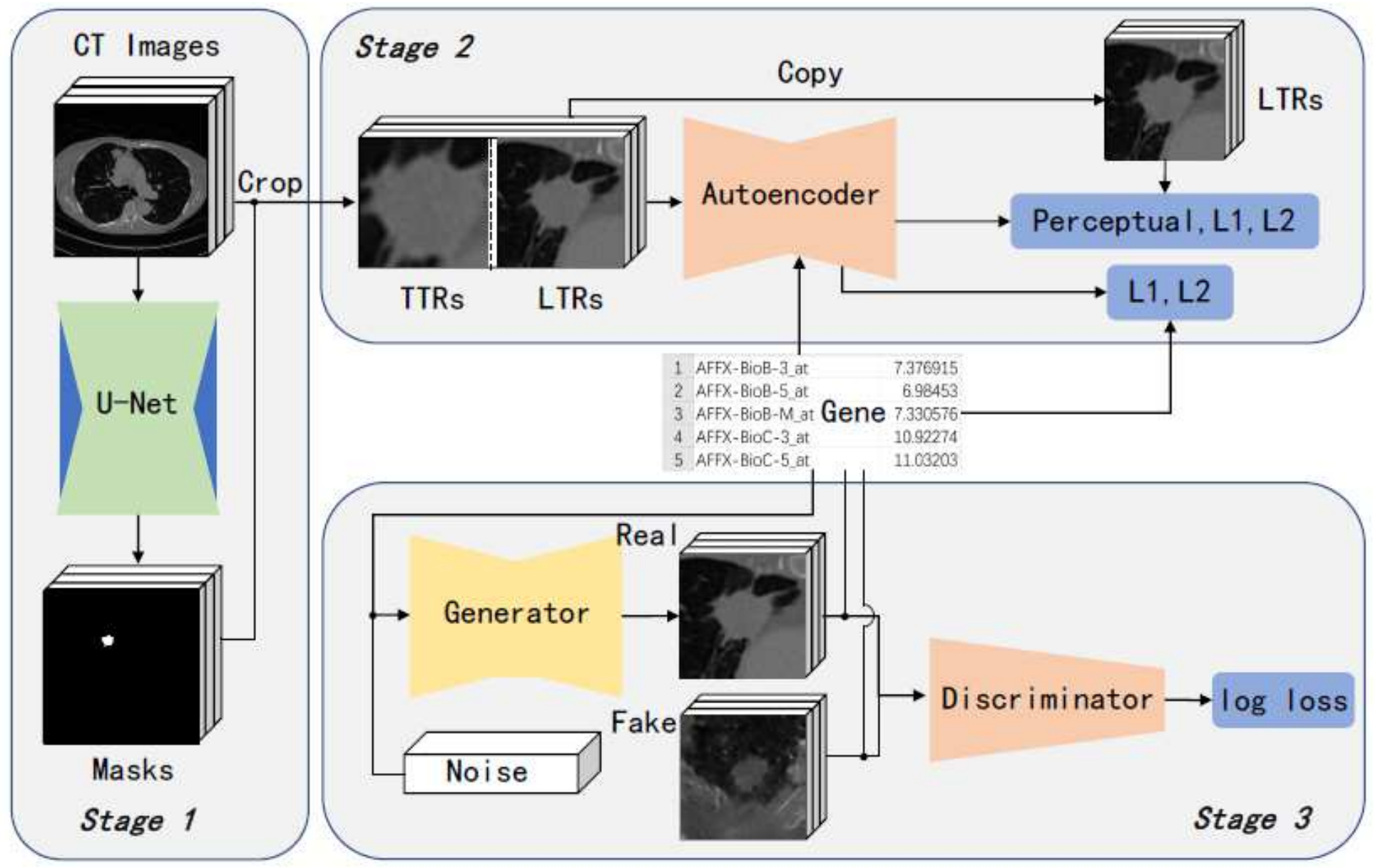

Figure 1

Work flow of our proposed framework. Stage 1: Tumor segmentation. Stage 2: Gene and image mapping. Stage 3: Synthetic tumor region generation.

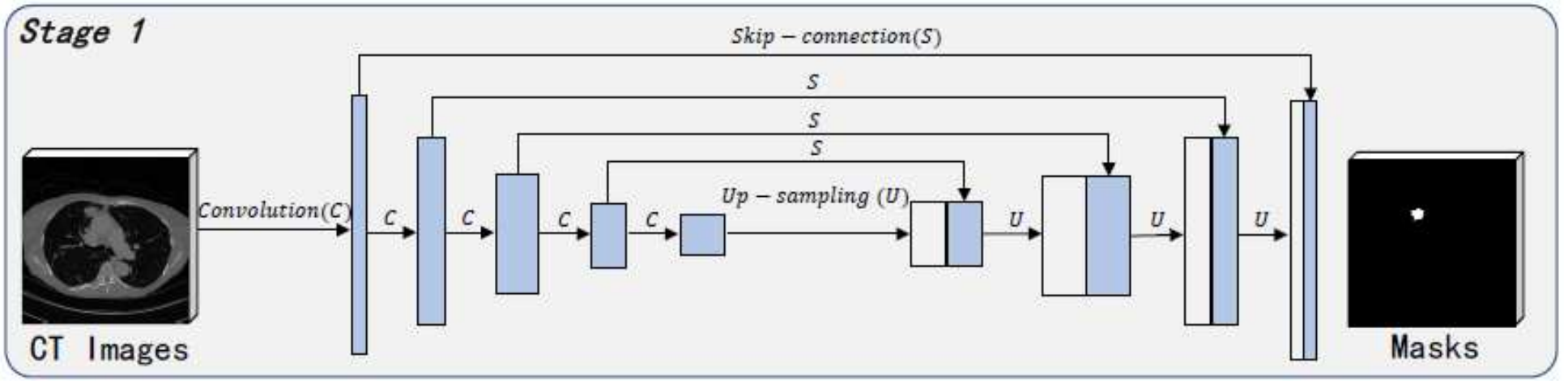

Figure 2

U-net architecture for tumor segmentation. 


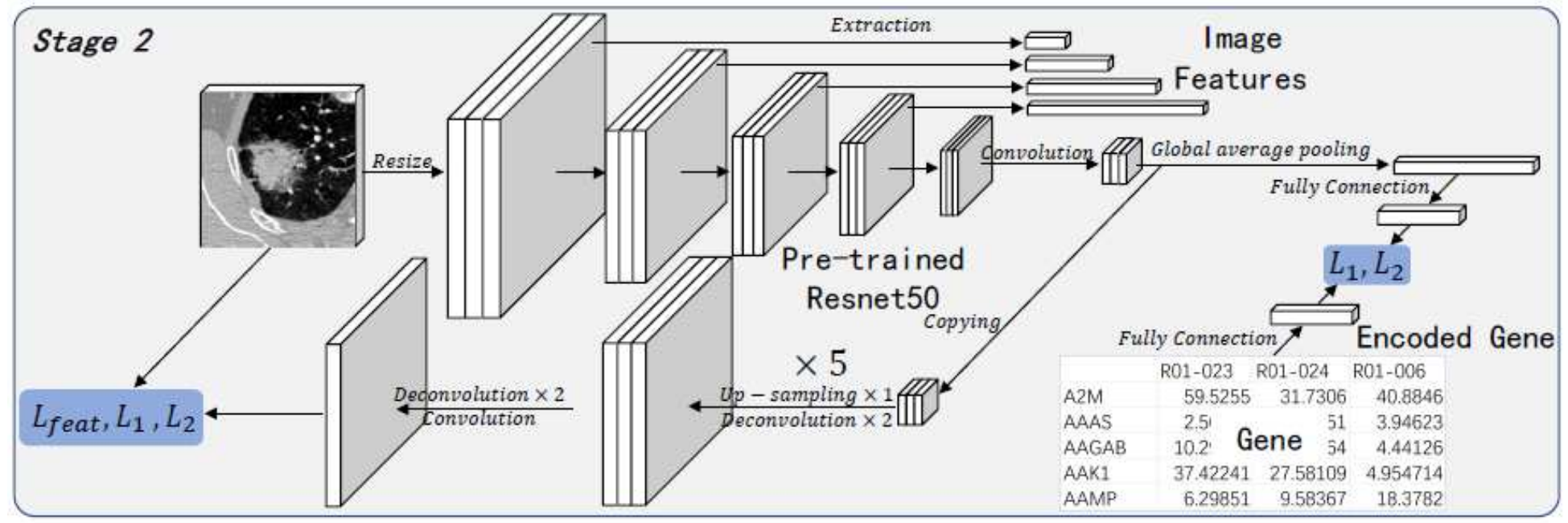

\section{Figure 3}

Illustration of GCVAE-GAN architecture. For better demonstrating this model, it is shown in 3D view. The erect labels are names of different modules and the italicized are operations between modules. The direction of arrows indicates the ow of data. Each sub-model is shown in different axis with name on the left $(E, G, D, C)$ and all six losses used in this model are connected by outputs of model and expectation.

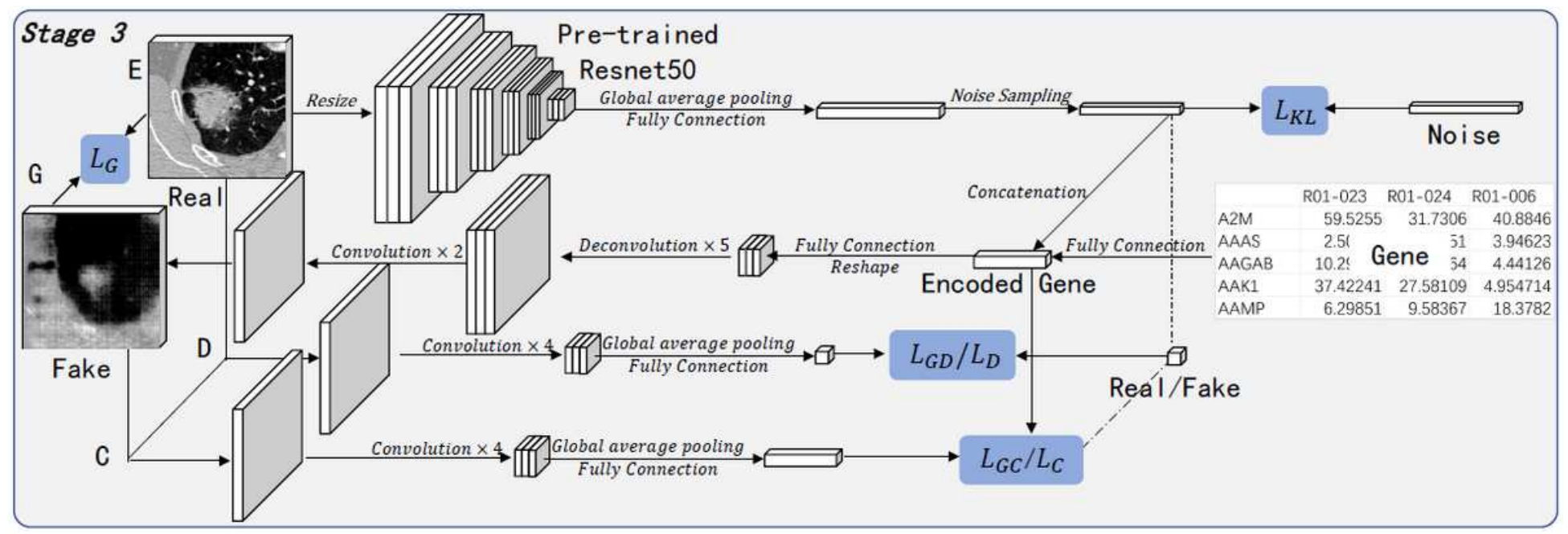

\section{Figure 4}

Illustration of GCVAE-GAN architecture. For better demonstrating this model, it is shown in 3D view. The erect labels are names of different modules and the italicized are operations between modules. The direction of arrows indicates the ow of data. Each sub-model is shown in different axis with name on the left $(E, G, D, C)$ and all six losses used in this model are connected by outputs of model and expectation. 


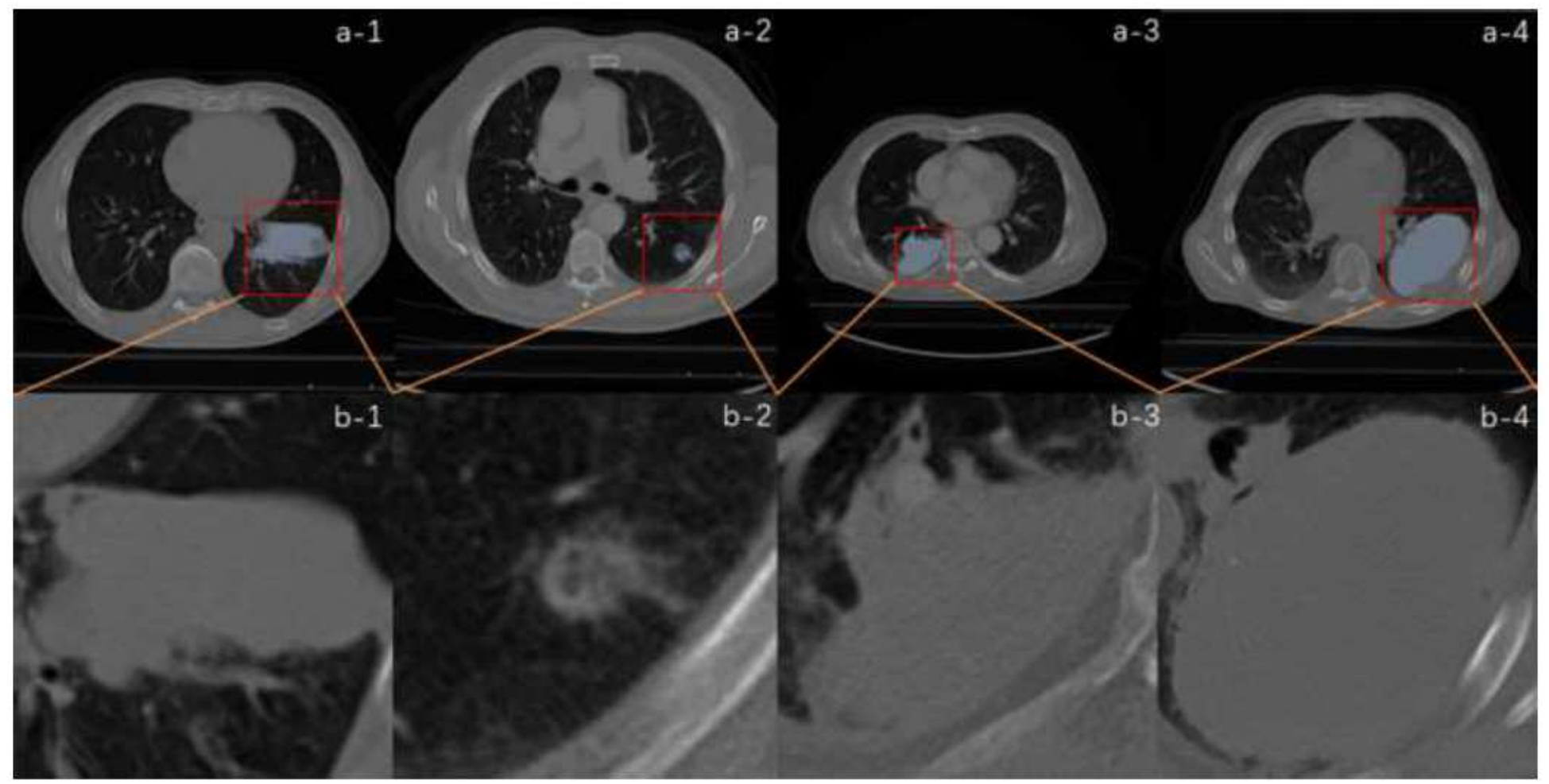

\section{Figure 5}

Illustration of our conditional autoencoder architecture. Images in row a are original CT images, tumors are labeled in red boundingboxes and the blue masks are results of segmentation. Images in row $b$ are cropped TRs with size of $128 \times 128$.

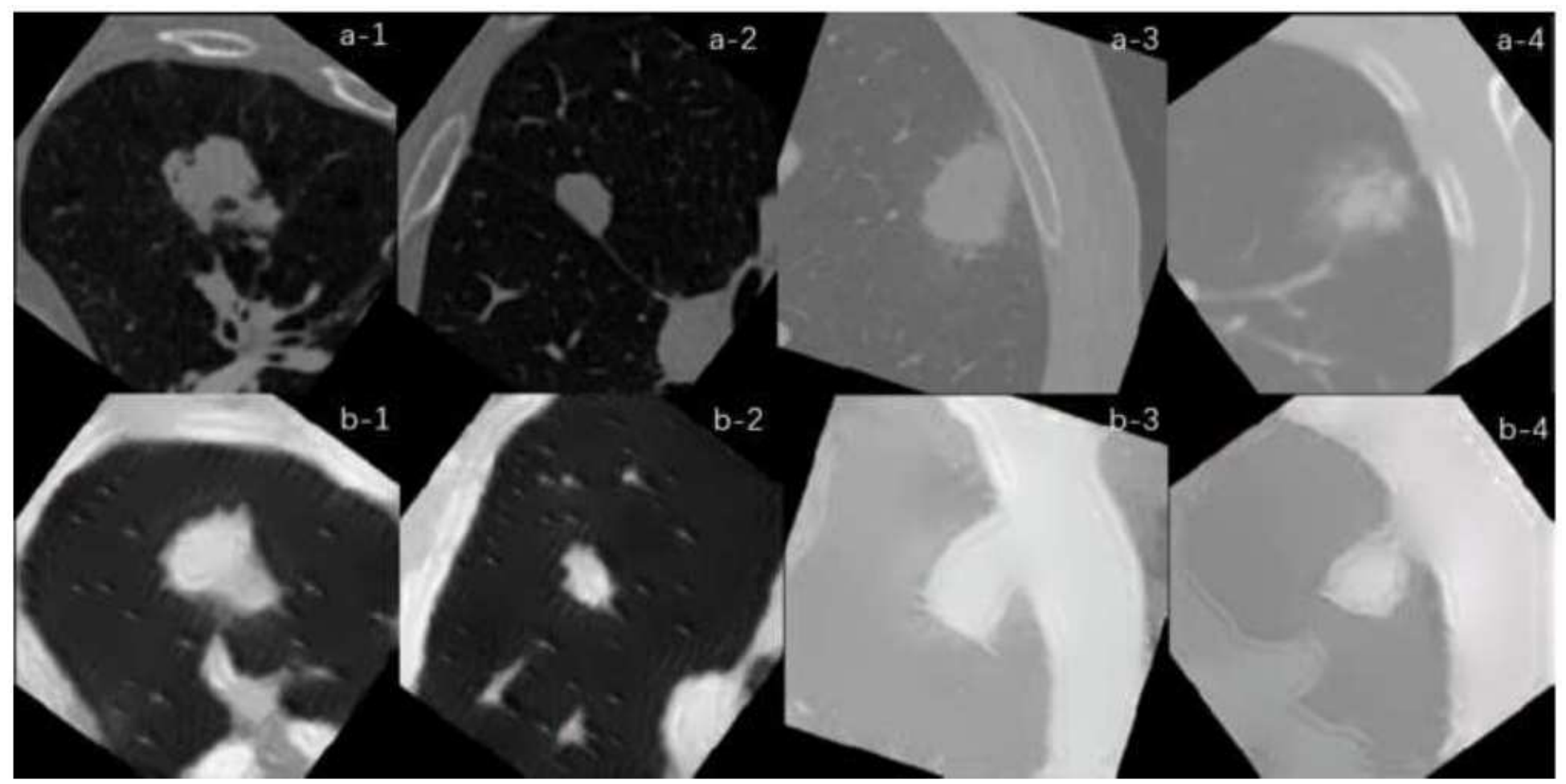

Figure 6 
The tumor images outputted by decoder in test set. Images in the row a are different original TRs, and images in row $b$ are corresponding synthetic TRs.

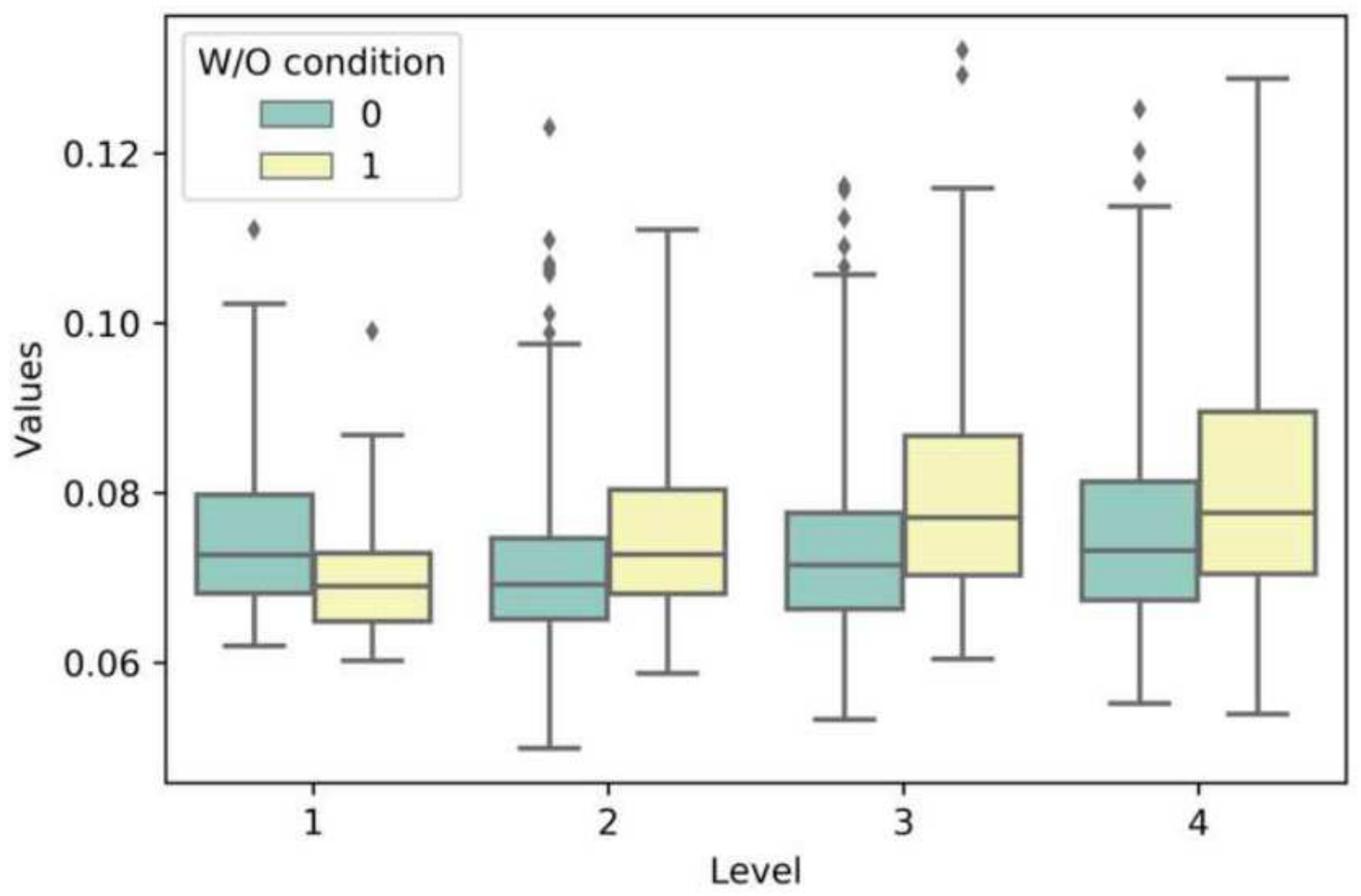

Figure 7

The difference of similarity with and without condition in each level. The similarity with condition is obviously higher than that without condition except level 1. 


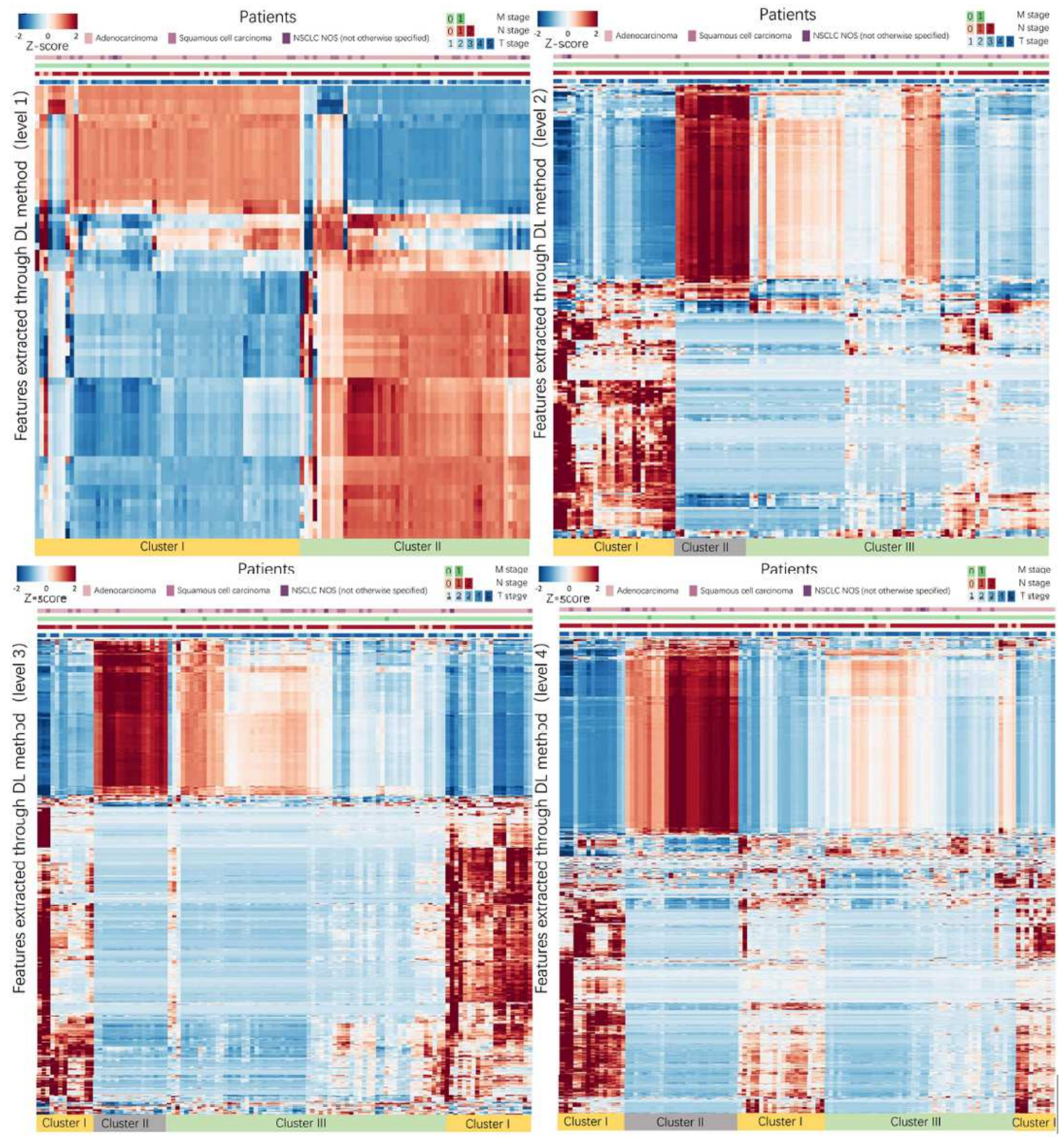

\section{Figure 8}

The difference of similarity with and without condition in each level. The similarity with condition is obviously higher than that without condition except level 1. 


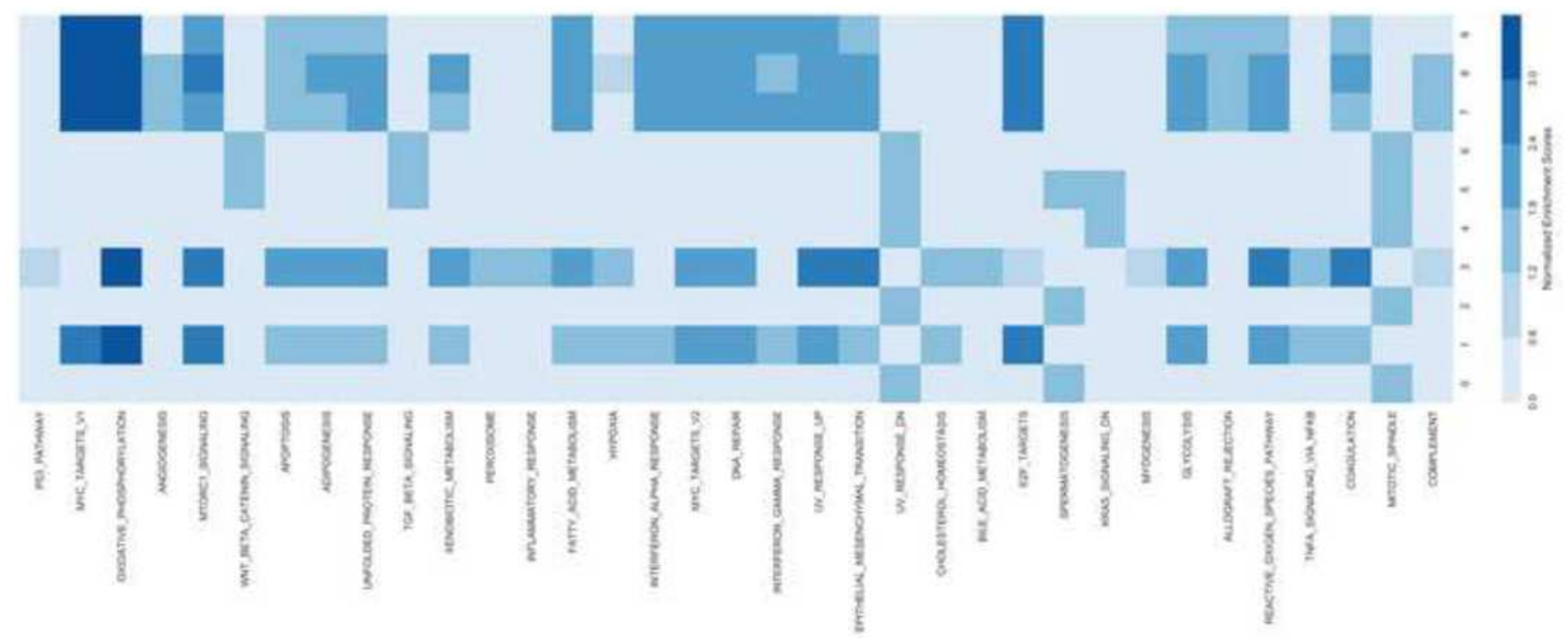

Figure 9

The correlation matrix between top ten DL features (level 4) related with all gene in each level and gene sets with high NES (NES $\geq 0.25$ ).
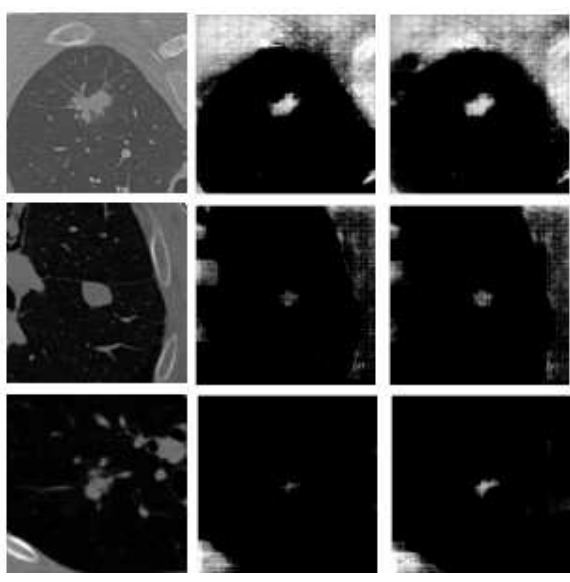
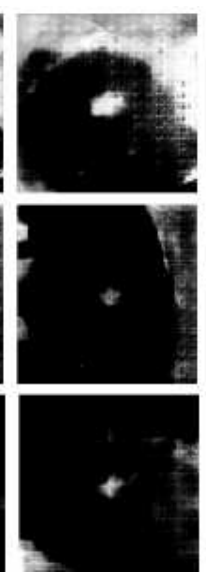
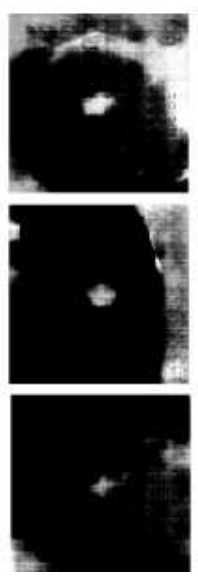
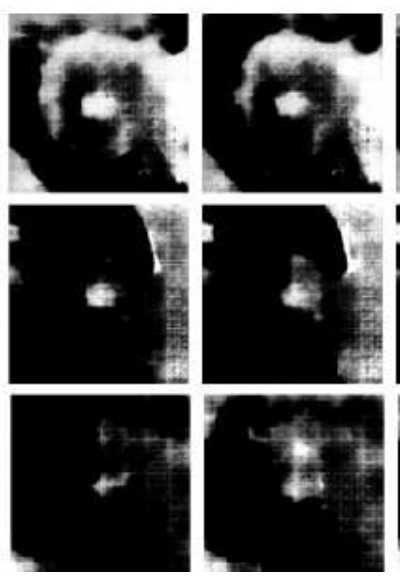
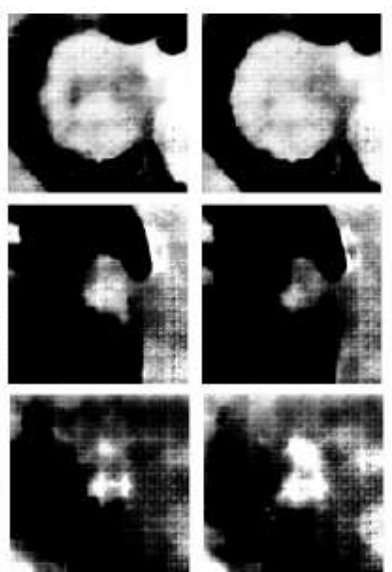
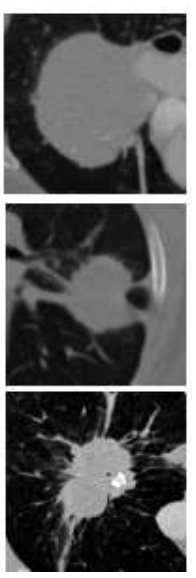

Figure 10

Tumor generation process while changing controllers gradually. The different rows are different samples. The first and last image in each row is real TR, and images between then are generated by "controller interpolation". 


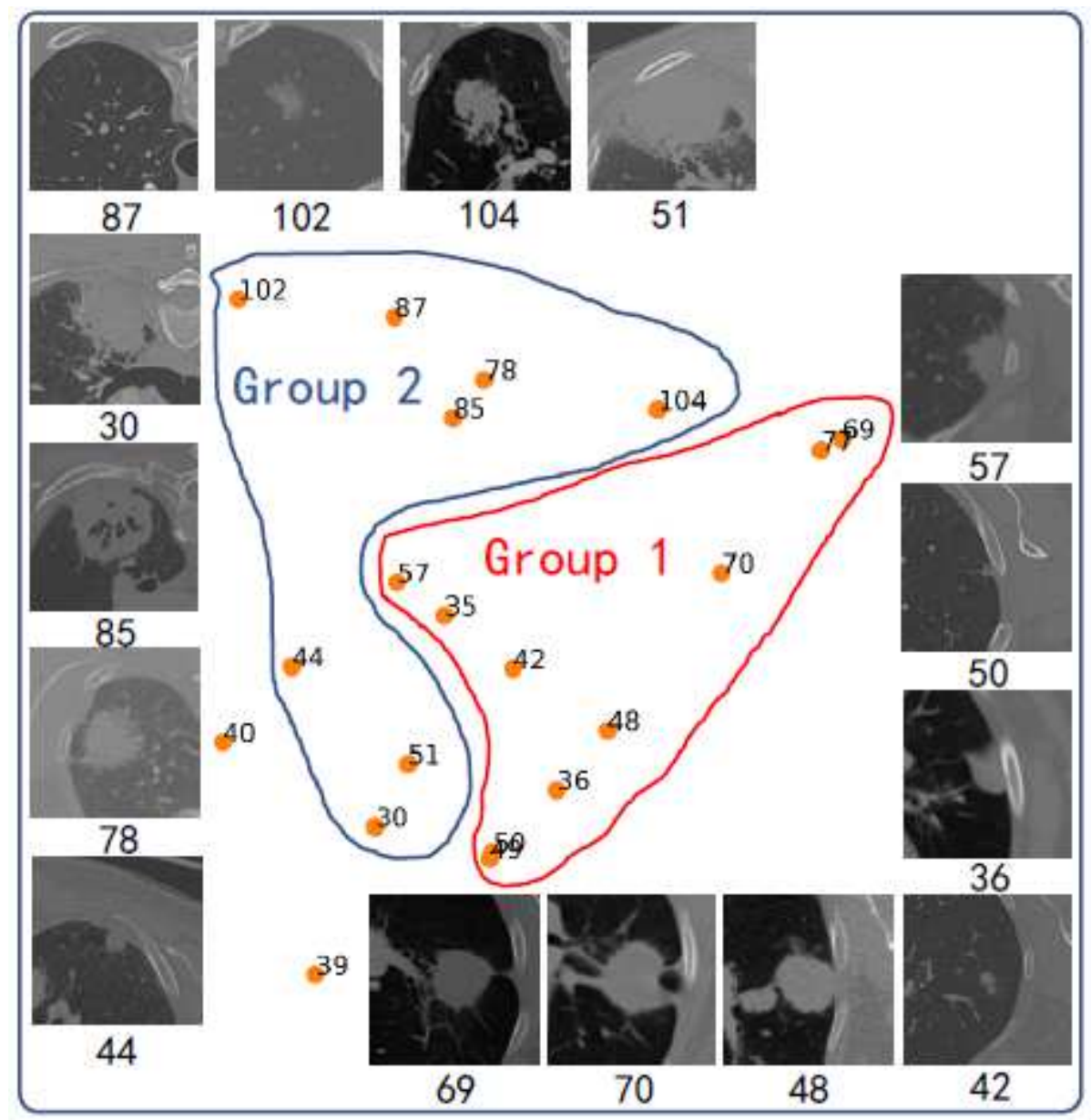

Figure 11

Part of samples' distribution on TSNE space. The numbers are index of samples. The samples in group 1 are all close to right edge of lung and those in group 2 are close to front of lung.

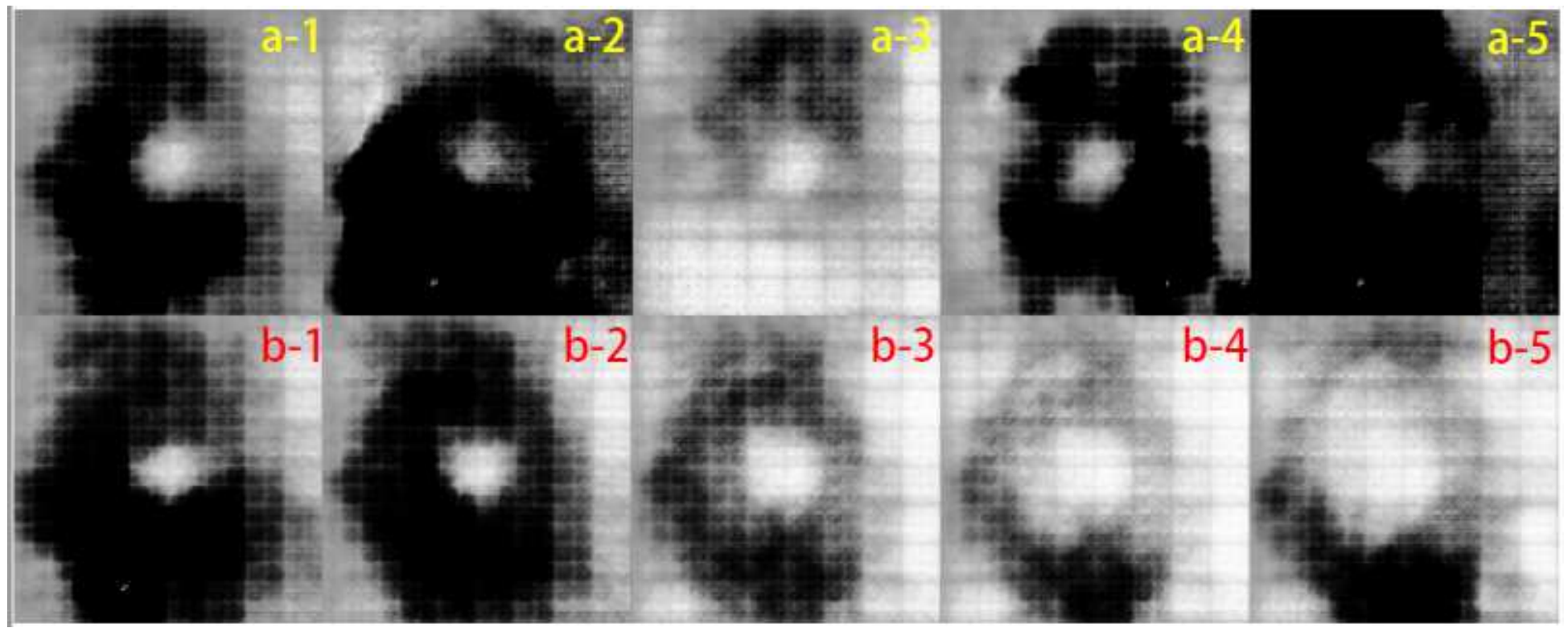

Figure 12 
Tumors generated using different location controllers (row a) and size controllers (row b). In row a, The first one $\mathrm{a}-1$ is the template, the others are tumors generated using activated front, behind, left, right controllers, respectively. In row b, The first one b- 1 is also the template, the others are generated with size controllers activated gradually.
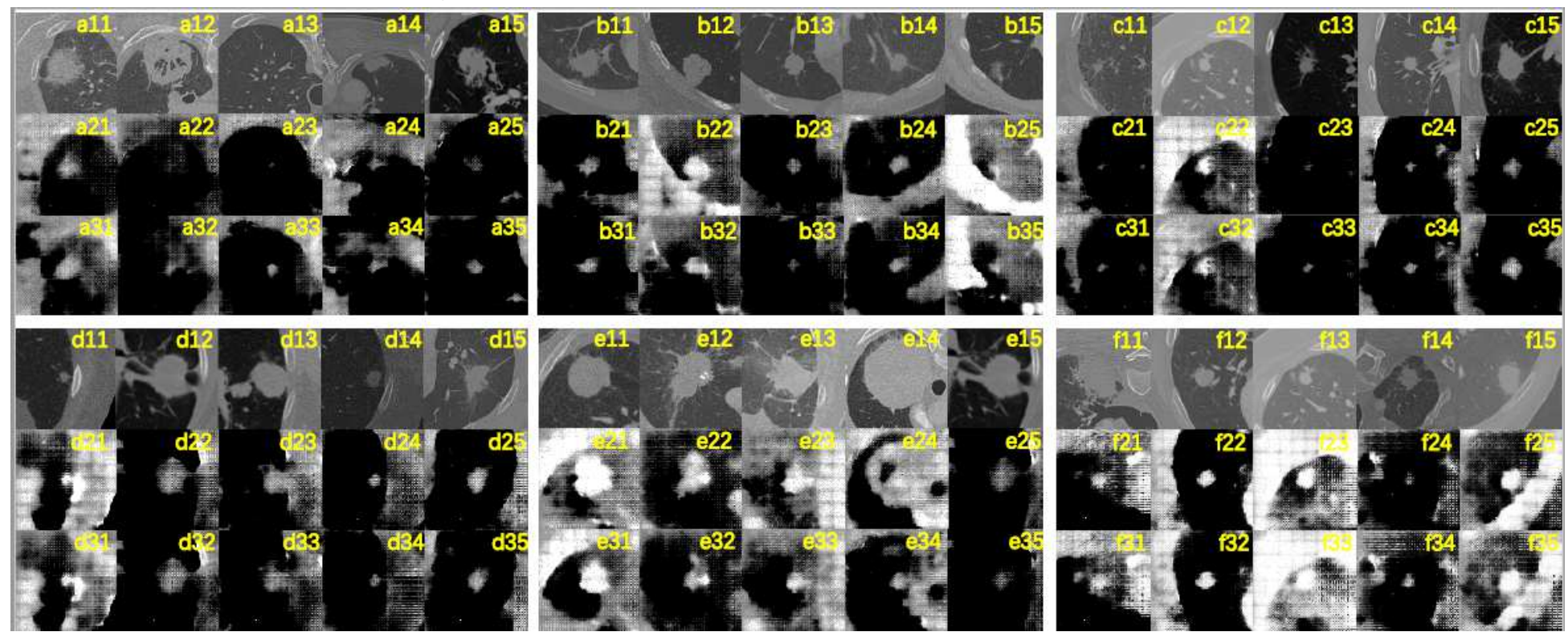

\section{Figure 13}

TRs generated by GCVAE-GAN with controllers activated or frozen. The images of group (a to d) are generated with corresponding controllers (front, behind, left, right) frozen. The images of group $(e, f)$ are generated with size controllers activated and frozen, respectively. In each group, images of first row are real TRs, images in second row are generated by corresponding genes which are controllers here, and images in third row are generated by corresponding switched controllers (frozen in (e) and activated in others). 